\title{
Competing for Customers in a Social Network (R)
}

\author{
Pradeep Dubey* $\quad$ Rahul Garg ${ }^{\dagger} \quad$ Bernard De Meyer $^{\ddagger}$
}

14 July $2013^{\S}$

\begin{abstract}
There are many situations in which a customer's proclivity to buy the product of any firm depends heavily on who else is buying the same product. We model these situations as non-cooperative games in which firms market their products to customers located in a "social network". Nash Equilibrium (NE) in pure strategies exist in general. In the quasi-linear version of the model, NE turn out to be unique and can be precisely characterized. If there are no a priori biases between customers and firms, then there is a cut-off level above which high cost firms are blockaded at an NE, while the rest compete uniformly throughout the network. Otherwise firms could end up as regional monopolies.

We also explore the relation between the connectivity of a customer and the money firms spend on him. This relation becomes particularly transparent when externalities are dominant: NE can be characterized in terms of the invariant measures on the recurrent classes of the Markov chain underlying the social network.

When we allow for cost functions of firms to be convex, instead of just linear, NE need no longer be unique as we show via an example. But uniqueness is restored if there is enough competition between firms or if their valuations of clients are anonymous.

Finally we develop a general model of nonlinear externalities and show that existence of NE remains intact.
\end{abstract}

\section{Introduction}

Consider a situation in which firms compete for customers located in a "social network". Any customer $i$ has, of course, a higher proclivity to buy from firm $\alpha$, if $\alpha$ spends more than its rivals on marketing its product to $i$. But another, quite independent, consideration also influences $i$ 's decision. He is keen to conform to his neighbors in the network. If the bulk of them purchase firm $\beta$ 's product, then he is tempted to do likewise. Such externalities arise naturally in several contexts and have become a subject of active exploration, especially from the viewpoint of their influence on both the marketing and the pricing strategies of firms (see, e.g., $[1,2,3,7,8,9,12,13,15,16,17])$. This paper is in the marketing framework.

It is evident that the externality in demand will have significant impact on the strategic interaction between the firms. Firm $\alpha$ may spend resources marketing its product to $i$, not because $\alpha$ cares about $i$ per se as a client, but because $i$ enjoys the position of a "hub" in the social network and so wields influence on other potential

*pradeepkdubey@yahoo.com, Center for Game Theory, Stony Brook University and Cowles Foundation, Yale University, USA

†rahul.garg@operasolutions.com, Opera Solutions, INDIA

‡demeyer@univ-paris1.fr, PSE-Univesité Paris 1, Paris, FRANCE

$\S$ This paper is an extension of [5].

The authors would like to thank two anonymous referees for extremely valuable suggestions. 
clients that are of value to $\alpha$. This in turn might instigate rival firms to spend further on $i$, since they wish to wean $i$ away from an excessive tilt toward $\alpha$; causing $\alpha$ to increase its outlay on $i$ even more, unleashing yet another round of incremental expenditures on $i$.

The scenario invites us to model it as a non-cooperative game between the firms. We take our cue from $[3,12]$ which explore the optimal marketing strategy of a single firm, based on the "network value" of the customers. (See also [9] for extensions and variants of [3, 12], developed from an algorithmic standpoint.) Our innovation is to introduce competition between several firms in this setting. The model we present is more general than that of $[3,12]$, though inspired by it. As in $[3,12]$, the social network, specifying the field of influence of each customer, is taken to be exogenous. Rival firms choose how much money to spend on each customer. For any profile of firms' strategies, the externality effect stabilizes over the social network and leads to unambiguous customer-purchases. This defines the non-cooperative game between firms that we explore here.

A prominent instance of our game arises when firms compete for advertisement space on different web-pages in the Internet (see Section 2.2). More generally our game pertains to a burgeoning class of industries in which firms compete for market share via strategies other than price variation. Think of new releases of movies or music albums. Here the prices are more or less the same across different products, and of little consequence for the consumer. The determining factor for him is his exposure to the product through advertisements; and, more importantly, the choices made by his "peer group" with whom he is eager to conform, including the critics whose reviews he values. Another example comes from telephony, where again the prices of different service providers are about the same (sometimes even controlled by a regulatory authority, as in India). But if most of the people that $i$ calls (i.e., $i$ 's neighbors in the social network) subscribe to service provider $\alpha$, and if $\alpha$-to- $\alpha$ calls have superior connectivity compared to $\alpha$-to- $\beta$ calls, then $i$ will have higher incentive to subscribe to $\alpha$ than to $\beta$. The reader can no doubt think of other products which are near-substitutes and whose sale prices have settled down to a small margin above cutthroat competitive costs; leaving firms to compete for clientele not by cutting prices, but via enhanced marketing strategies. Indeed, the growth of internet has engendered fierce competition in many product markets, so that only those firms survive which quote rockbottom prices. The competition between the survivors then happens mostly on the marketing plane. Of course this is not to say that there are not other important industries in which the competition between firms is centered on setting prices, taking the social network into account. There is an important strand of literature dealing with this (see in particular $[6,13,2]$ for monopoly pricing, $[1,2,8]$ for oligopolistic price competition, and the references therein). The model we present here should be viewed as complementary to that strand.

Our main interest is in understanding the structure of the NE of the game between the firms. Will they end up as regional monopolies, operating in separate parts of the network? Or will they compete fiercely throughout? Which firms will enter the fray, and which will be blockaded? And how will the money spent on a customer depend on his connectivity in the social network?

Section 2 introduces the quasi-linear model (and includes those in [3], [12] by setting \# firms =1). We show that $\mathrm{NE}$ are unique and can be easily computed in polynomial time via closed-form expressions involving matrix inverses. It turns out that, provided that there are no a priori biases between firms and customers, any NE has a cut-off cost: all firms whose costs are above the cut-off are blockaded, and the rest enter the fray. Moreover there is no "regionalization" of firms in an NE: each active firm spends money on every customer-node of the social network. The money spent on node $i$ is related to the connectivity of $i$, but the relation is somewhat subtle,

${ }^{1}$ We also show that a priori biases can lead to regional monopolies among the firms.(See Section 3.3.) 
though expressible in precise algebraic form. When externalities are dominant ${ }^{2}$, however, this relation becomes more transparent: NE can be characterized in terms of the invariant measures on the recurrent classes of the Markov chain underlying the social network (see Section 3.4). In particular suppose that the graph representing the social network is undirected and connected, all the neighbors of any customer-node exert equal influence on him, and each company values all the nodes equally. Then, at the NE, the money spent by a company on a node is proportional to the degree of the node.

In Section 4 we consider convex (rather than linear) cost functions, which include the fixed-budget case where each firm can spend freely up to an exogenously specified limit. NE need not be unique as we show via an example in Section 4.2. But if there is "sufficient competition", in that each firm has enough rivals whose characteristics are nearby, then the uniqueness of NE is restored (see Section 4.4). Uniqueness also holds if firms' valuation of clients are anonymous (see Section 4.5), no matter how heterogenous their costs.

In Section 5 we describe a general non-linear model. So long as the externalities are a contraction, the strategy-to-outcome map (and thus the game) is well-defined. We then show that NE exist in pure strategies under the standard convexity assumptions.

For the convenience of the reader most proofs have been put out of the way, in an appendix. The only exception is Theorem 3, whose proof comes to hand in the course of developing the model.

\section{The Quasi-Linear Model}

\subsection{The Model}

There are finite sets $\mathcal{A}$ of firms and $\mathcal{I}$ of customers. We shall define a strategic game $\Gamma$ among the firms. The customers themselves are non-strategic in our model and described in behavioristic terms, as in [3, 12].

Firm $\alpha \in \mathcal{A}$ can spend $m_{i}^{\alpha}$ dollars on customer $i \in \mathcal{I}$ by way of marketing its product to him. The strategy set of firm $\alpha$ may thus be viewed $\operatorname{as}^{3} R_{+}^{\mathcal{I}}$, with elements $m^{\alpha}:=\left(m_{i}^{\alpha}\right)_{i \in \mathcal{I}}$, representing $\alpha^{\prime}$ s expenditures across customers in $\mathcal{I}$.

Consider a profile of firms' strategies $m:=\left(m^{\beta}\right)_{\beta \in \mathcal{A}} \in R_{+}^{\mathcal{I} \times \mathcal{A}}$. The proclivity of customer $i$ to buy from any particular firm $\alpha$ clearly depends on the profile $m$, i.e., not just the expenditure of $\alpha$ but also those of its rivals. We denote this proclivity by $p_{i}^{\alpha}(m)$. One can think of $p_{i}^{\alpha}(m)$ as the quantity of $\alpha$ 's product purchased by $i$. Or, interpreting $i$ to be a mass of customers ${ }^{4}$ such as those who visit a web page $i$, one can think of $p_{i}^{\alpha}(m)$ as the fraction of mass $i$ that goes to $\alpha$ or, equivalently, as the probability of $i$ going to $\alpha$ (recall, different companies $\alpha$ are vying for customers on any given webpage $i$ ). In either setting, we take $p_{i}(m):=\left(p_{i}^{\beta}(m)\right)_{\beta \in \mathcal{A}} \in[0,1]^{\mathcal{A}}$. (When $p_{i}^{\alpha}(m)$ is a quantity, there is an implicit upper bound on customer $i$ 's capacity to consume which, w.l.o.g., is normalized to be 1.)

The benefit to any particular firm $\alpha$ from its clientele $p^{\alpha}(m):=\left(p_{i}^{\alpha}(m)\right)_{i \in \mathcal{I}}$ is $\sum_{i \in \mathcal{I}} u_{i}^{\alpha} p_{i}^{\alpha}(m)$ and the cost of its expenditures $m^{\alpha}$ is $\sum_{i \in \mathcal{I}} c_{i}^{\alpha} m_{i}^{\alpha}$. We assume that

$$
\text { for all } \alpha \text { and all } i: c_{i}^{\alpha}>0 \text { and } u_{i}^{\alpha} \geq 0, u^{\alpha} \neq 0
$$

(The condition $u^{\alpha} \neq 0$ is without loss of generality, since $\alpha$ would always chose $m^{\alpha}=0$, i.e., "not play the game" if $u^{\alpha}=0$, and therefore could be dropped from $\mathcal{A}$.)

\footnotetext{
${ }^{2}$ At the other extreme, where externalities are absent, our model yields the much-studied Tullock contest [18].

${ }^{3}$ or, more generally, a convex subset of $R_{+}^{\mathcal{I}}$.

${ }^{4}$ Such an interpretation of $i$ as a mass is also given in [1]
} 
Then $\alpha$ 's payoff in the game is given by

$$
\Pi^{\alpha}(m)=\sum_{i \in \mathcal{I}} u_{i}^{\alpha} p_{i}^{\alpha}(m)-\sum_{i \in \mathcal{I}} c_{i}^{\alpha} m_{i}^{\alpha}
$$

It remains to define the map from $m$ to $p(m)$.

Customer $i$ 's proclivity $p_{i}^{\alpha}$ to purchase from firm $\alpha$ is clearly positively correlated with $\alpha$ 's expenditure $m_{i}^{\alpha}$ on $i$, and negatively correlated with the expenditures $m_{i}^{-\alpha}:=\left(m_{i}^{\beta}\right)_{\beta \in \mathcal{I} \backslash\{\alpha\}}$, of $\alpha$ 's rivals (since we are thinking here of firms' products as substitutes).

In addition we suppose that there is a positive externality exerted on $i$ by the choice of any neighbor $j$ : increases in $p_{j}^{\alpha}$ may boost $p_{i}^{\alpha}$. Cross-effects of $p_{j}^{\beta}$ on $p_{i}^{\alpha}$, for $\beta \neq \alpha$, can be incorporated under certain assumptions (see Section 5.1), but to begin with we shall suppose for simplicity that they are absent.

To define the map from $m$ to $p(m)$, we must take full cognizance of the social network. In section 5 , we consider a general mathematical framework which allows for nonlinear network effects, but for the moment let us restrict to the linear case. In this event, the social network may be represented by a finite, directed, weighted graph $G=(\mathcal{I}, E, w)$. The nodes of $G$ are identified with the set of customers $\mathcal{I}$. Each directed edge $(i, j) \in E:=\mathcal{I} \times \mathcal{I}$ has weights $\left(w_{i j}^{\alpha}\right)_{\alpha \in \mathcal{A}}$, where $w_{i j}^{\alpha} \geq 0$ is a measure of the influence $j$ has on $i$, with regard to purchases from $\alpha$. Precisely, if $p^{\alpha}=\left(p_{j}^{\alpha}\right)_{j \in \mathcal{I}}$ denotes the proclivities of purchases from $\alpha$, then the externality impact $^{5}$ of $p^{\alpha}$ on $i$ is $\sum_{j \in \mathcal{I}} w_{i j}^{\alpha} p_{j}^{\alpha}$. We assume that $\sum_{j \in \mathcal{I}} w_{i j}^{\alpha} \leq 1$, for all $i \in \mathcal{I}$ and $\alpha \in \mathcal{A}$. (One may view $\left(\mathcal{I}, E^{\alpha}, w^{\alpha}\right)$ as the social network relevant for firm $\alpha$, with $\left.E^{\alpha}=\left\{(i, j) \in E: w_{i j}^{\alpha}>0\right\}\right)$.

Let us now make explicit how firms' expenditures, in conjunction with the externality effect, determine purchases in the social network.

Fix a profile $m:=\left(m^{\beta}\right)_{\beta \in \mathcal{A}}:=\left(\left(m_{j}^{\beta}\right)_{j \in \mathcal{I}}\right)_{\beta \in \mathcal{A}}$ of firms' strategies. Denote by $m_{i}=\left(m_{i}^{\beta}\right)_{\beta \in \mathcal{A}}$ the vector of expenditures on $i$ by all the firms when $m$ is played.

For any firm $\alpha$ and customer $i$, let $\gamma_{i}^{\alpha}\left(m_{i}\right) \in[0,1]$ denote the direct marketing impact of $m_{i}$ on $\alpha$ 's proclivity $p_{i}^{\alpha}$ to buy from firm $\alpha$. Thus $\gamma_{i}^{\alpha}$ is a map from $R_{+}^{\mathcal{A}}$ to $[0,1]$

Denote $\sum_{\beta \in \mathcal{A}} m_{i}^{\beta}$ by $\bar{m}_{i}$. Our key assumption on $\gamma_{i}^{\alpha}\left(m_{i}\right)$ is that it depends only on the variables $m_{i}^{\alpha}$ and $\bar{m}_{i}{ }^{-\alpha}:=\sum_{\beta \in \mathcal{A} \backslash\{\alpha\}} m_{i}^{\beta}$, i.e., firm $\alpha$ is affected only by the aggregate ${ }^{6}$ expenditure of its rivals. We shall abuse notation slightly and write $\gamma_{i}^{\alpha}\left(m_{i}\right)=\gamma_{i}^{\alpha}\left(m_{i}^{\alpha}, \bar{m}_{i}^{-\alpha}\right)$.

AI For $m_{i} \neq 0: \gamma_{i}^{\alpha}$ is continuous in all its variables and, for any fixed $\bar{m}_{i}^{-\alpha}$, it is nondecreasing, differentiable and concave in $m_{i}^{\alpha}$ (concavity reflecting the diminishing returns to $\alpha$ of incremental dollars spent on $i$ ).

The key examples we have in mind are the functions $\gamma_{i}^{\alpha}$ defined as follows (for any fixed $r$ and $d$ in $[0,1)$ ):

$$
\gamma_{i}^{\alpha}\left(m_{i}\right)= \begin{cases}\left(m_{i}^{\alpha} / \bar{m}_{i}\right)\left(\bar{m}_{i}\right)^{r} & \text { if } \bar{m}_{i}>0 \\ d & \text { if } \bar{m}_{i}=0\end{cases}
$$

where $\bar{m}_{i}:=\left(\sum_{\beta \in \mathcal{I}} m_{i}^{\beta}\right)$. In short, $i$ 's probability of purchase from different firms is simply set proportional to the money that firms spend on him if $\bar{m}_{i}>0$, and is arbitrary if $\bar{m}_{i}=0$. (As will become clear, the NE will not be affected by the choice of $d$, for any given $r$.) We may think of $\left(\bar{m}_{i}\right)^{r}$ as the "market penetration", which

\footnotetext{
${ }^{5}$ Strictly speaking the term $\sum_{j \in \mathcal{I} \backslash\{i\}} w_{i j}^{\alpha}$ embodies the "pure" externality effect of others on $i$; but we allow for $w_{i i}^{\alpha}>0$.

${ }^{6}$ Aggregation is a form of anonymity that is common to many markets. It says, in essence, that if a firm pretends to be two entities and splits its expenditure between them, this has no effect on other firms. This form of "anonymity toward numbers" is tantamount to aggregation.
} 
rises with the total money spent. (If $\gamma_{i}^{\alpha}\left(m_{i}\right)$ is to be a probability, one must amend $\left(\bar{m}_{i}\right)^{r}$ to $\max \left\{\left(\bar{m}_{i}^{r}\right), 1\right\}$ or a suitably smoothed version of this function.)

When $r=0$, we shall call it the canonical example. This is a special case of a Tullock contest [18].

Our last assumption has to do with the possible discontinuity of the function $\gamma_{i}^{\alpha}\left(m_{i}\right)$ as $m_{i} \rightarrow 0$. Indeed in our canonical example there is an irremovable discontinuity at 0: the limit of $\gamma_{i}^{\alpha}\left(m_{i}\right)$ can be any number between 0 and 1 depending on the direction from which $m_{i}$ approaches 0 . To steer clear of 0 , we introduce AII below. The idea is that, for each customer $i$, there be at least two distinct firms who value $i$, so that the competition between them will ensure that the total money spent on $i$ is positive in any NE. The intuition is that, if $m_{i}$ is too small, either of the two firms could spend a "sliver" $\delta>0$ on $i$, which costs very little, but is nevertheless overwhelmingly more than other firms' expenditures on $i$, and thus is able to "buy out" $i$, contradicting that it has optimized. Precisely we have

AII For all $i \in \mathcal{I}$, there is a set $\mathcal{A}_{i} \subset \mathcal{A}$ of at least two firms such that

(1) For all $\alpha \in \mathcal{A}_{i}, u_{i}^{\alpha}>0$.

(2) For all $i \in \mathcal{I}$, for all $\alpha \in \mathcal{A}_{i}$, for all sequences $\left\{m_{k}\right\}_{k \in N} \subset \mathcal{M}$, with $m_{k}=\left(m_{k, i}^{\alpha}\right)_{i \in \mathcal{I}, \alpha \in \mathcal{A}}$, such that $m_{k, i}^{\alpha} \leq \bar{m}_{k, i} / 2$ for all $k$ and $\bar{m}_{k, i} \rightarrow 0$ as $k \rightarrow \infty$.

$$
\lim _{\delta \rightarrow 0}\left\{\limsup _{k \rightarrow \infty}\left[\gamma_{i}^{\alpha}\left(m_{k, i}^{\alpha}+\delta, m_{k, i}^{-\alpha}\right)-\gamma_{i}^{\alpha}\left(m_{k, i}^{\alpha}, m_{k, i}^{-\alpha}\right)\right] / \delta\right\}=\infty
$$

(3) For all $i \in \mathcal{I}$ and for all $\alpha \in \mathcal{A}_{i}, \lim _{\epsilon \rightarrow 0}\left[\gamma_{i}^{\alpha}(\epsilon, 0)-\gamma_{i}^{\alpha}(0,0)\right] / \epsilon=\infty$.

To interpret (2) of AII , consider a unilateral deviation by $\alpha$ wherein $\alpha$ increases $m_{k, i}^{\alpha}$ to $m_{k, i}^{\alpha}+\delta$ for fixed $\delta$. Since all $\beta \in \mathcal{A} \backslash\{\alpha\}$ have expenditures $m_{k, i}^{\beta}$ on $i$ that become vanishingly small compared to the expenditure $m_{k, i}^{\alpha}+\delta$ made by $\alpha$, as $k$ goes to $\infty$, firm $\alpha$ must have $100 \%$ of the "marketing impact" on $i$ in the limit, on account of its deviation. On the other hand, it has less than $50 \%$ of the impact, prior to its deviation, since its expenditure is over-matched by at least one rival firm. But the jump from $50 \%$ to $100 \%$ is non-negligible since - as will be made precise shortly - $i$ is not guided solely by the externality effect of his neighbors, but puts positive weight on the marketing impact $\gamma_{i}^{\alpha}$. The boost in $\gamma_{i}^{\alpha}$ is given by the bracketed term [...] in (2) of AII which is at least of the order of $1 / 2$ and so the whole term goes to infinity like $1 /(2 \epsilon)$ as $\epsilon \rightarrow 0$. Our assumption is somewhat weaker since it imposes no constraint on the rate at which the bracketed term goes to $\infty$.

As for (3) of AII , the intuition is that if there is no firm pursuing customer $i$, then there is a huge "firstmover" advantage to firm $\alpha$ when it alone approaches $i$ : for an infinitesimal expenditure of $\epsilon$ dollars, $\alpha$ can trigger a disproportionately large sale of $L(\epsilon)$ dollars to $i$, where $L(\epsilon) / \epsilon \rightarrow \infty$ as $\epsilon \rightarrow 0$.

It is easy to see that our key examples satisfy both (2) and (3) of AII .

Turning to behavior of customer $i$ in our model, he weights the two factors (i.e., the externality impact and the marketing impact) by $\theta_{i}^{\alpha}$ and $1-\theta_{i}^{\alpha}$, where $0 \leq \theta_{i}^{\alpha}<1$. Thus, given a strategy profile $m$, the final steady-state proclivities of purchase $p(m):=\left(p^{\alpha}(m)\right)_{\alpha \in \mathcal{A}} \in[0,1]^{\mathcal{I} \times \mathcal{A}}$, where $p^{\alpha}(m):=\left(p_{j}^{\alpha}(m)\right)_{j \in \mathcal{I}}$, must satisfy.

$$
p_{i}^{\alpha}(m)=\left(1-\theta_{i}^{\alpha}\right) \gamma_{i}^{\alpha}\left(m_{i}\right)+\theta_{i}^{\alpha} \sum_{j \in \mathcal{I}} w_{i j}^{\alpha} p_{j}^{\alpha}(m)
$$

for all $\alpha \in \mathcal{A}$ and $i \in \mathcal{I}$. 
Define the $|\mathcal{I}| \times|\mathcal{I}|$-matrices: $I:=$ identity, $\Theta^{\alpha}:=$ the diagonal matrix with $\Theta_{i i}^{\alpha}=\theta_{i}^{\alpha}$ and $W^{\alpha}:=$ the matrix with entries $w_{i j}^{\alpha}$. Then equation (4) reads

$$
p^{\alpha}(m)=\left(I-\Theta^{\alpha}\right) \gamma^{\alpha}(m)+\Theta^{\alpha} W^{\alpha} p^{\alpha}(m) .
$$

Since $I-\Theta^{\alpha} W^{\alpha}$ is invertible ${ }^{7}$ (the row sums of $\Theta^{\alpha} W^{\alpha}$ being less than 1 ), we obtain

$$
p^{\alpha}(m)=\left(I-\Theta^{\alpha} W^{\alpha}\right)^{-1}\left(I-\Theta^{\alpha}\right) \gamma^{\alpha}(m) .
$$

This gives (see (2)) firm $\alpha$ 's payoff

$$
\Pi^{\alpha}(m)=\left[u^{\alpha}\right]^{\top}\left(I-\Theta^{\alpha} W^{\alpha}\right)^{-1}\left(I-\Theta^{\alpha}\right) \gamma^{\alpha}(m)-\left[c^{\alpha}\right]^{\top} m^{\alpha}
$$

where $u^{\alpha}:=\left(u_{j}^{\alpha}\right)_{j \in \mathcal{I}} \in R_{+}^{\mathcal{I}}$ and $c^{\alpha}:=\left(c_{j}^{\alpha}\right)_{j \in \mathcal{I}} \in R_{++}^{\mathcal{I}}$ are column vectors and $T$ stands for the transpose operation.

This completes the definition of the strategic game.

Recall that a strategy profile $m$ is called an $\mathrm{NE}^{8}$ of this game if

$$
\Pi^{\alpha}(m) \geq \Pi^{\alpha}\left(\tilde{m}^{\alpha}, m^{-\alpha}\right) \quad \forall \tilde{m}^{\alpha} \in R_{+}^{\mathcal{I}}
$$

for all $\alpha \in \mathcal{A}$ (where $\left.m^{-\alpha}:=\left(m^{\beta}\right)_{\beta \in \mathcal{I} \backslash\{\alpha\}}\right)$.

Denote

$$
v^{\alpha}:=\left[u^{\alpha}\right]^{\top}\left(I-\Theta^{\alpha} W^{\alpha}\right)^{-1}\left(I-\Theta^{\alpha}\right)
$$

Then (5) may be rewritten:

$$
\Pi^{\alpha}(m)=\sum_{i \in \mathcal{I}}\left(v_{i}^{\alpha} \gamma_{i}^{\alpha}\left(m_{i}\right)-c_{i}^{\alpha} m_{i}^{\alpha}\right)
$$

Denote

$$
\phi_{i}^{\alpha}\left(m_{i}^{\alpha}, \bar{m}_{i}^{-\alpha}\right):=\frac{\partial}{\partial m_{i}^{\alpha}} \gamma_{i}^{\alpha}\left(m_{i}^{\alpha}, \bar{m}_{i}^{-\alpha}\right)
$$

and next define

$$
\lambda_{i}^{\alpha}\left(r_{i}^{\alpha}, \bar{m}_{i}\right):=\phi_{i}^{\alpha}\left(r_{i}^{\alpha} \bar{m}_{i},\left(1-r_{i}^{\alpha}\right) \bar{m}_{i}\right)
$$

(Thus $r_{i}^{\alpha}:=m_{i}^{\alpha} / \bar{m}_{i}$.) We suppose that

AIII For $\bar{m}_{i}>0, \lambda_{i}^{\alpha}$ is strictly decreasing in $r_{i}^{\alpha}$ (for fixed $\bar{m}_{i}$ ); and non-increasing in $\bar{m}_{i}$ (for fixed $r_{i}^{\alpha}$ ).

The assumption AIII also reflects the diminishing returns on incremental dollars spent by $\alpha$; it further states that an incremental dollar of $\alpha$ counts for less when $\alpha$ 's rivals have put in more money.

It is easy to check that assumption AIII is also satisfied by our key examples.

This completes the formal description of the quasi-linear model. We conclude this section by presenting a concrete application of this model.

\footnotetext{
${ }^{7}$ The "diagonal dominance" property for $I-\Theta W$ can clearly be replaced by the weaker technical condition that all the eigenvalues of $\Theta W$ have a modulus strictly less than one. From the modeling point of view, however, the sub-stochasticity assumption on $W$ is easier to test, and seems more natural (see our example on advertising on the web, where $W$ is even stochastic).

${ }^{8}$ Throughout we confine attention to "pure" strategies.
} 


\subsection{An Application: Competition for Advertisement on the Web.}

Think of the web as a set $\mathcal{I}$ of pages, each of which corresponds to a distinct node of a graph. A directed arc $(i, j)$ means that there is a link from page $j$ to page $i$.

At the beginning of any period, two kind of "surfers" visit page $i$. There are those who transit to $i$ from other pages $j$ in the web. Furthermore, there are "fresh arrivals", entering the web for the first time, via page $i$ at rate $\psi_{i}$.

At the end of the period, a fraction $\left(1-\theta_{i}\right)$ of the population on the page $i$ exits the web, while the remaining fraction $\theta_{i}$ continues surfing (where $0 \leq \theta_{i}<1$ ). The weight on $(i, j)$, which we denote $w_{i j}$, gives the probability that a representative surfer, who is on page $j$ and who continues surfing, moves on to page $i$ (or, alternatively, the fraction of surfers on page $j$ who transit to page $i$ ). Thus $\sum_{i \in \mathcal{I}} w_{i j}=1$ for all $j \in \mathcal{I}$.

Companies $\alpha \in \mathcal{A}$ compete for advertisement on the web pages. If they spend $m_{i}:=\left(m_{i}^{\alpha}\right)_{\alpha \in \mathcal{A}}$ dollars to place their advertisements on page $i$, they get "visibility" (time, space) on page $i$ in proportion to the money spent. Thus the probability that a surfer views company $\alpha$ 's advertisement on page $i$ is $m_{i}^{\alpha} / \bar{m}_{i}=\gamma_{i}^{\alpha}\left(m_{i}^{\alpha}, \bar{m}_{i}^{-\alpha}\right)$.

The payoff of a company is the aggregate "eyeballs" of its advertisement obtained, in the long run (i.e., in the steady state).

To compute the payoff, let us first examine the population distribution of surfers across nodes in the unique steady state of the system.

Denote by $\zeta_{i}$ the arrival rate of surfers (of both kinds) to page $i$. Then, in a steady state, we must have

$$
\zeta_{i}=\psi_{i}+\sum_{j \in \mathcal{I}} w_{i j} \theta_{j} \zeta_{j}
$$

for all $i \in \mathcal{I}$. In matrix notation, this is

$$
\zeta=\psi+W \Theta \zeta
$$

where $\zeta:=\left(\zeta_{i}\right)_{i \in \mathcal{I}}$ and $\psi:=\left(\psi_{i}\right)_{i \in \mathcal{I}}$ are column vectors, $\Theta$ is the diagonal $\mathcal{I} \times \mathcal{I}$ matrix with entries $\theta_{i i}=\theta_{i}$, and $W$ is the $\mathcal{I} \times \mathcal{I}$ matrix with entries $w_{i j}$. Hence

$$
\zeta=(I-W \Theta)^{-1} \psi
$$

The total eyeballs (per period) obtained by company $\alpha$ is then

$$
\sum_{i \in \mathcal{I}} \zeta_{i} \gamma_{i}^{\alpha}(m)
$$

which fits the format of (7).

More generally, suppose surfers have bounded recall of length $k$. Then firm $\alpha$ will only care about any surfer's eyeballs in the last $k$ periods prior to the surfer's exit. When $k=1, \alpha$ 's payoff is

$$
\sum_{i \in \mathcal{I}}\left(1-\theta_{i}\right) \zeta_{i} \gamma_{i}^{\alpha}(m)
$$

The expression for $v_{i}^{\alpha}$ will become complicated when the recall $k>1$ (more so, if discounting of past memory is incorporated). But the payoffs in all these cases still fit the format of (7).

Generalizing in a different direction, suppose that surfers at page $i$, who have spent $t$ periods in the web, exit at rate $\theta_{i}^{t}$ for $t=1,2 \ldots$. Denote by $\Theta^{t}$ the diagonal matrix whose $i i^{t h}$ entry is $\theta_{i}^{t}$. Then $\zeta=\left(I+W \Theta^{1}+\right.$ $\left.W \Theta^{2} W \Theta^{1}+\ldots\right) \psi$, which is well-defined provided we assume $\theta_{i}^{t} \leq \Delta<1$ for some $\Delta$ (for all $t, i$ ). This retains 
the format of (7) though the expression for $v_{i}^{\alpha}$ becomes even more complicated. One could also incorporate bounded recall in this setting, without departing from (7).

Notice that the "externality" in the above examples is reflected in the movement of traffic across pages in the web. Also notice that the games derived are anonymous i.e. $v_{i}^{\alpha}=v_{i}$ for all $\alpha$. Such games will be singled out for special attention later.

\section{Nash Equilibrium and its Characterization}

\subsection{Existence and Uniqueness of Pure-Strategy Nash Equilibria}

Theorem 1 Under assumptions AI and AII there exists an NE in the quasi-linear model. Moreover if $m$ is an $N E$, then $\bar{m}_{i}>0$, for all $i \in \mathcal{I}$. Finally, if AIII also holds, the $N E$ is unique.

\subsection{General Structure}

Theorem 2 Consider our canonical case: $\gamma_{i}^{\alpha}\left(m_{i}\right)=m_{i}^{\alpha} / \bar{m}_{i}$ (other closed-form expressions for the $\gamma_{i}^{\alpha}$ will lead to analogous characterizations). Fix customer $i$ and rank all the firms in $\mathcal{A}:=\{1,2, \ldots, n\}$ in order of increasing $\kappa_{i}^{\alpha}:=c_{i}^{\alpha} / v_{i}^{\alpha}$ (see (6) for the definition of $v_{i}^{\alpha}$ ). For convenience denote this order $\kappa_{i}^{1} \leq \kappa_{i}^{2} \leq \ldots \leq \kappa_{i}^{n}$. Let

$$
k_{i}=\max \left\{l \in\{2, \ldots, n\}:(l-2) \kappa_{i}^{l}<\sum_{\alpha=1}^{l-1} \kappa_{i}^{\alpha}\right\}
$$

In the unique $N E$, firms $1, \ldots, k_{i}$ will spend money on customer $i$ as follows:

$$
m_{i}^{\alpha}=\left(\frac{k_{i}-1}{\sum_{\beta=1}^{k_{i}} \kappa_{i}^{\beta}}\right)\left(1-\frac{\left(k_{i}-1\right) \kappa_{i}^{\alpha}}{\sum_{\beta=1}^{k_{i}} \kappa_{i}^{\beta}}\right)
$$

Firms $k_{i}+1, \ldots, n$ put no money on customer $i$.

According to Theorem 2, companies $\alpha$ can be ranked, at each customer-node $i$, according to their "effective costs" $\kappa_{i}^{\alpha}$. The money $m_{i}^{\alpha}$, spent by $\alpha$ on $i$, is a strictly decreasing function of $\kappa_{i}^{\alpha}$ up to some threshold, after which it becomes zero.

Theorem 2 confirms the obvious intuition that $m_{i}^{\alpha}=0$ if $v_{i}^{\alpha}=0$ (i.e., $\kappa_{i}^{\alpha}=\infty$, recalling that $c_{i}^{\alpha}>0$ by assumption). It also brings to light a different, and more important, feature of NE. First recall that, by (6), $v_{i}^{\alpha}$ may well be highly positive even though the direct value $u_{i}^{\alpha}$ of customer $i$ to company $\alpha$ is zero. This is because $v_{i}^{\alpha}$ incorporates the network value of $i$, stemming from the possibility that $i$ may be exerting a big externality on other customers whom $\alpha$ does directly value. Now, since $\kappa_{i}^{\alpha}$ falls as $v_{i}^{\alpha}$ increases, (10) reveals that $\alpha$ may be spending a huge $m_{i}^{\alpha}$ on $i$ even when $u_{i}^{\alpha}$ is zero, purely on account of the network value of $i$.

\subsection{Regionalized versus Unified Customer Territory}

Suppose there are five customers $\{1,2, \ldots, 5\}$ and four firms $\left\{\alpha_{1}, \alpha_{2}, \beta_{1}, \beta_{2}\right\}$. The customers are arranged in a linear network, with $i$ connected to $i+1$ via an undirected (i.e., directed both ways) edge, for $i=$ $1,2,3,4$. Suppose each node is equally influenced by its neighbors in the purchase of any firm's product. Thus $\left(w_{11}^{\gamma}, w_{12}^{\gamma}, w_{13}^{\gamma}, w_{14}^{\gamma}, w_{15}^{\gamma}\right)=(0,1,0,0,0),\left(w_{21}^{\gamma}, w_{22}^{\gamma}, w_{23}^{\gamma}, w_{24}^{\gamma}, w_{25}^{\gamma}\right)=(0.5,0,0.5,0,0)$ etc., for any company 
$\gamma$. Further suppose $\theta_{i}^{\gamma}=0.1$ and $c_{i}^{\gamma}=1$ for all $\gamma$ and $i$. Finally let $u^{\alpha_{1}}=u^{\alpha_{2}}=(1,1,0,0.1,0.1)$ and $u^{\beta_{1}}=u^{\beta_{2}}=(0.1,0.1,0,1,1)$. Formula (6) yields $v^{\alpha_{1}}=v^{\alpha_{2}}=(0.950,0.998,0.055,0.102,0.095)$ and $v^{\beta_{1}}=$ $v^{\beta_{2}}=(0.095,0.102,0.055,0.998,0.950)$ and hence $\kappa^{\alpha_{1}}=\kappa^{\alpha_{2}}=(1.053,1.002,18.182,9.779,10.514)$ and $\kappa^{\beta_{1}}=$ $\kappa^{\beta_{2}}=(10.514,9.779,18.182,1.002,1.053)$. It follows from Theorem 2 that firms $\alpha_{1}$ and $\alpha_{2}$ will put no money on customers 4,5 and positive money on the rest; while firms $\beta_{1}$ and $\beta_{2}$ will put no money on customers 1,2 and positive money on the rest. In effect, there will "regionalization" of customers into $\alpha$-territory $\{1,2,3\}$ and $\beta$-territory $\{3,4,5\}$. The only overlap is customer 3 , who is of zero direct value $u_{3}^{\gamma}$ to all firms $\gamma$ and yet is being equally targeted by them, purely on account of his network value.

The situation dramatically changes when the game is anonymous i.e., $v_{i}^{\alpha}=v_{i}$ and $c_{i}^{\alpha}=c^{\alpha}$ for all $\alpha$ and $i$. (The first identity holds in particular - see $(6)-$ when $w_{i j}^{\alpha}=w_{i j}, \theta_{i}^{\alpha}=\theta_{i}$, and $u_{i}^{\alpha}=u_{i}$, for all $\alpha, i$ and $j$, i.e., there are no a priori biases between firms and customers.) Our analysis in Section 3.2 immediately implies that we can rank the firms, independently of $i$, by their costs; say (after relabeling)

$$
c^{1} \leq c^{2} \leq \ldots \leq c^{n}
$$

At the NE a subset of low-cost firms $\{1, \ldots, k\}$ will be active (see (9)), while all the higher-cost firms $\{k+1, \ldots, n\}$ will be blockaded, where

$$
k=\max \left\{l \in\{2, \ldots, n\}:(l-2) c^{l}<\sum_{\beta=1}^{l-1} c^{\beta}\right\}
$$

Each active firm $\alpha \in\{1, \ldots, k\}$ will spend an amount $m_{i}^{\alpha}>0$ on all the nodes $i \in \mathcal{I}$ that is proportional to $v_{i}$. Indeed, by (10), we have

$$
m_{i}^{\alpha}=\frac{v_{i}(k-1)}{\sum_{\beta=1}^{k} c^{\beta}}\left(1-\frac{(k-1) c^{\alpha}}{\sum_{\beta=1}^{k} c^{\beta}}\right)
$$

which also shows that $\bar{m}^{\alpha} \geq \bar{m}^{\beta}$ if $\alpha<\beta$, i.e., lower cost firms spend more money than their higher-cost rivals. Finally, adding (11) over $\alpha$, we have

$$
\bar{m}_{i}=\frac{v_{i}(k-1)}{\sum_{\beta=1}^{k} c^{\beta}}
$$

which means that the money spent on customer $i$ is proportional to his network value $v_{i}$. In contrast to our first example, there is nothing akin to regionalization of customer territory at NE. Instead, firms that are not blockaded, compete uniformly throughout the social network.

In the special case of complete symmetry among the firms (i.e., we also have $c_{i}^{\alpha}=c$ for all $\alpha \in \mathcal{A}$ ), all the firms will be active and the above equations will hold with $k=n$.

\subsection{When Externalities become Dominant: A Markov Chain Perspective}

It is often is too expensive for a firm $\alpha$ to provide significant subsidies $m_{i}^{\alpha}$ to each customer $i$. Indeed the marketing division of firm $\alpha$ is typically allocated a fixed budget $M^{\alpha}$ and, if there is a large population of customers, then the individual expenditures $m_{i}^{\alpha}$ must perforce be small. In this event, customers' behavior is predominantly driven by the externality effect of their neighbors. We can capture the situation in our model by supposing that all the $\theta_{i}^{\alpha}$ are close to 1 . 
Thus we are led to inquire about the limit of the NE as the $\theta_{i}^{\alpha} \longrightarrow 1$ for all $\alpha$ and $i$. (In this scenario we will also obtain a more transparent relation between NE and the graphical structure of the social network.)

To this end - and even otherwise - it is useful to recast our model in probabilistic terms. Assume, for simplicity, that $\sum_{j \in \mathcal{I}} w_{i j}^{\alpha}=1$ for all $i$ and $\alpha$. Let us consider a Markov chain with $\mathcal{I}$ as the state space and $W^{\alpha}$ as the transition matrix (i.e., $w_{i j}^{\alpha}$ is the probability of going from $i$ to $j$ ). Let $i_{t}$ denote the (random) state of the chain at date $t=0,1,2, \ldots$. Suppose that, upon arrival in state $i_{t}$, a choice $L_{t} \in\{$ Stop, Move $\}$ is made with $\operatorname{Prob}\left(L_{t}=\right.$ Move $)=\theta_{i_{t}}^{\alpha}$. Let $T$ be the first time $L_{t}=S$ top and consider the random variable $\gamma_{i_{T}}^{\alpha}(m)$. If $\rho^{\alpha}(i)$ denotes the conditional expectation $E\left[\gamma_{i_{T}}^{\alpha}(m) \mid i_{0}=i\right]$, then clearly the $I$-dimensional vector $\rho^{\alpha}$, substituted for $p^{\alpha}(m)$, satisfies equation (4). Since this equation has a unique solution, it must be the case that $p^{\alpha}(m)=\rho^{\alpha}$.

Recall that by (1) each vector $u^{\alpha}$ is nonnegative and nonzero, and so we may write $u^{\alpha}=y^{\alpha} \xi^{\alpha}$, where $y^{\alpha}>0$ is a scalar and $\xi^{\alpha}$ is a probability distribution on $\mathcal{I}$. The weighted sum $\left[u^{\alpha}\right]^{\top} p(m)$ is then equal to $y^{\alpha} \sum_{i \in \mathcal{I}} \xi_{i}^{\alpha} \rho^{\alpha}(i)$ which in turn can be expressed as $y^{\alpha} E\left[\gamma_{i_{T}}^{\alpha}(m)\right]$, provided we assume that the probability distribution of the initial state $i_{0}$ is $\xi^{\alpha}$. Therefore the vector $v^{\alpha} / y^{\alpha}$ is just the probability distribution of $i_{T}$ initializing the Markov chain at $\xi^{\alpha}$.

We want to analyze the asymptotic behavior of $v^{\alpha}$ as the $\theta_{i}^{\alpha}$ converge to 1 (since the unique NE of our games are determined by $v^{\alpha}$ ). Let us first consider the simple case when $\theta_{i}^{\alpha}=\theta^{\alpha}$ for all $i$. Then the random time $T$ becomes independent of the Markov chain and we get easily that $\operatorname{prob}(T=t)=\left(1-\theta^{\alpha}\right)\left(\theta^{\alpha}\right)^{t}$.

Therefore

$$
\begin{aligned}
v_{i}^{\alpha} / y^{\alpha} & =\operatorname{prob}\left(i_{T}=i\right) \\
& =\sum_{t=0}^{\infty} \operatorname{prob}(T=t) \operatorname{prob}\left(i_{t}=i \mid T=t\right) \\
& =\sum_{t=0}^{\infty} \operatorname{prob}(T=t) \operatorname{prob}\left(i_{t}=i\right) \\
& =\sum_{t=0}^{\infty} \operatorname{prob}(T=t) E\left[\mathbb{1}_{i}\left(i_{t}\right)\right] \\
& =E\left[\sum_{t=0}^{\infty}\left(1-\theta^{\alpha}\right)\left(\theta^{\alpha}\right)^{t} \mathbb{1}_{i}\left(i_{t}\right)\right]
\end{aligned}
$$

where $\mathbb{1}_{i}$ is the indicator function of $i: \mathbb{1}_{i}(j)=0$ if $j \neq i$ and $\mathbb{1}_{i}(i)=1$.

Recall that a sequence $\left\{a_{t}\right\}_{t \in \mathbb{N}}$ of real numbers is said to

i) Abel -converge to $a$ if $\lim _{\theta \rightarrow 1} \sum_{t=0}^{\infty}(1-\theta)(\theta)^{t} a_{t}=a$.

ii) Cesaro-converge to $a$ if $\lim _{N \rightarrow \infty} N^{-1} \sum_{t=0}^{N-1} a_{t}=a$.

The Frobenius Theorem (see, e.g., line 11 on page 65 of [10]) states that a Cesaro-convergent sequence is Abel-convergent to the same limit. So, to analyze the limit behavior of $v_{i}^{\alpha}$, it is sufficient to consider the Cesaro-convergence of $\left\{\mathbb{1}_{i}\left(i_{t}\right)\right\}_{t \in \mathbb{N}}$.

The finite state-set $\mathcal{I}$ of our Markov chain can be partitioned into recurrent classes $I_{1}^{\alpha}, \ldots, I_{k(\alpha)}^{\alpha}$ and a set of transient states $I_{0}^{\alpha}$. Each recurrent class $I_{s}^{\alpha}$ is the support of a unique invariant probability measure $\mu_{s}^{\alpha}$ (see, e.g., Theorem 2.4 in chapter $\mathrm{V}$ of [4]).

If the Markov process starts within a recurrent class $I_{s}^{\alpha}$ (i.e., $i_{0} \in I_{s}^{\alpha}$ ), then the ergodic Theorem states that, for an arbitrary function $f$ on $\mathcal{I}, N^{-1} \sum_{t=0}^{N-1} f\left(i_{t}\right)$ converges almost surely to $E_{\mu_{s}^{\alpha}}[f]$.

If it starts at a transient state $i \in I_{0}^{\alpha}$, then we may define the first time $\tau$ that it enters $\cup_{s \geq 1} I_{s}^{\alpha}$. Let $S$ be the index of the recurrence class $i_{\tau}$ belongs to. The ergodic Theorem also tells us in this case that $N^{-1} \sum_{t=0}^{N-1} f\left(i_{t}\right)$ converges almost surely to the random variable $E_{\mu_{S}^{\alpha}}[f]$.

Let us define $\hat{\mu}^{\alpha, i}$ as the expectation $E\left[\mu_{S}^{\alpha}\right]$, if $i \in I_{0}^{\alpha}$ and as $\mu_{s}^{\alpha}$ if $i \in I_{s}^{\alpha}(s \geq 1)$. Then we clearly get $E\left[N^{-1} \sum_{t=0}^{N-1} f\left(i_{t}\right) \mid i_{0}=i\right] \longrightarrow E_{\hat{\mu}^{\alpha, i}}[f]$. Therefore, denoting $\hat{\mu}^{\alpha}:=\sum_{i \in \mathcal{I}} \xi_{i}^{\alpha} \hat{\mu}^{\alpha, i}$, and invoking the Frobenius Theorem, we have proved: 
Theorem 3 As $\theta^{\alpha}$ tends to 1 , $v_{i}^{\alpha}$ converges to $y^{\alpha} E_{\hat{\mu}^{\alpha}}\left[\mathbb{1}_{i}\right]=y^{\alpha} \hat{\mu}_{i}^{\alpha}$.

Corollary 1 Suppose that the graph of the underlying social network is undirected and connected. Further suppose

$$
\theta_{i}^{\alpha}=\theta, w_{i k^{\prime}}=w_{i k} \text { and } \sum_{j \in \mathcal{I}} w_{i j}=1
$$

for all $\alpha \in \mathcal{A}, i \in \mathcal{I}$ and $k, k^{\prime}$ such that $w_{i k}>0$ and $w_{i k^{\prime}}>0$ (i.e., all the nodes connected to $i$ have the same influence on $i)$. Finally suppose that $u_{i}^{\alpha}$ is invariant of $i$ for all $\alpha$ (i.e., each company values all clients equally), w.l.o.g. $u_{i}^{\alpha}=1 /|\mathcal{I}|$ for all $\alpha$ and $i$. Then as $\theta$ tends to 1 , the money spent at $N E$ by a company on any node is proportional to the degree of the node.

Proof: Let $E$ be the set of edges $(i, j)$ in the graph. $E$ is a symmetric set because the graph is undirected. We then have $w_{i, j}=\mathbb{1}_{(i, j) \in E} / N_{i}$, where $N_{i}:=\sum_{j} \mathbb{1}_{(i, j) \in E}$. Since the graph is also connected, the Markov chain is irreducible and thus has a unique invariant measure $\mu$ that must satisfy the equation $\mu^{\top} W=\mu^{\top}$. Now observe that $D=\left(D_{i}\right)_{i \in \mathcal{I}}$, with $D_{i}:=\sum_{k} \mathbb{1}_{(k, i) \in E}$ denoting the degree of node $i$, also satisfies equation $D^{\top} W=D^{\top}$. Indeed

$$
\begin{aligned}
\sum_{i} D_{i} w_{i, j} & =\sum_{i, k} \mathbb{1}_{(k, i) \in E} \mathbb{1}_{(i, j) \in E} / N_{i} \\
& =\sum_{i, k} \mathbb{1}_{(i, k) \in E} \mathbb{1}_{(i, j) \in E} / N_{i} \\
& =\sum_{i} \mathbb{1}_{(i, j) \in E}\left(\sum_{k} \mathbb{1}_{(i, k) \in E}\right) / N_{i} \\
& =\sum_{i} \mathbb{1}_{(i, j) \in E} \\
& =D_{j},
\end{aligned}
$$

where the second inequality comes from the symmetry of $E$, and the following ones from the definition of $N_{i}$ and $D_{i}$.

We infer that the invariant measure is proportional to the degree (i.e. $\mu=\lambda D$.). By Theorem $3, v_{i}^{\alpha}=v_{i}$ converges to the degree of $i$ as $\theta$ tends to 1 . But, by equation (11) in Section 3.3, $m_{i}^{\alpha}$ is proportional to $v_{i}$.

It might be useful to illustrate Theorem 3 with a simple example. Consider the network with four nodes, corresponding to the following matrix $W^{\alpha}$ for firm $\alpha$ :

$$
W^{\alpha}=\left(\begin{array}{cccc}
0 & .5 & .5 & 0 \\
.5 & 0 & 0 & .5 \\
0 & 0 & 1 & 0 \\
0 & 0 & 0 & 1
\end{array}\right) .
$$

Also let $u_{1}^{\alpha}=7, u_{2}^{\alpha}=8, u_{3}^{\alpha}=10, u_{4}^{\alpha}=11$. Nodes 3 and 4 are clearly absorbent: once reached by the process, they become permanent. Nodes 1 and 2 are transient. Hence $I_{0}^{\alpha}=\{1,2\}, I_{1}^{\alpha}=\{3\}$ and $I_{2}^{\alpha}=\{4\}$.

The invariant probability measure $\mu_{1}^{\alpha}$ (resp. $\mu_{2}^{\alpha}$ ) places all the weight on node 3 (resp. 4). Therefore,

$$
\hat{\mu}^{\alpha, 3}=\mu_{1}^{\alpha}=(0,0,1,0) \text { and } \hat{\mu}^{\alpha, 4}=\mu_{2}^{\alpha}=(0,0,0,1)
$$

Observing that $\operatorname{prob}\left(i_{t+1} \in\{3,4\} \mid i_{t}=i\right)=1 / 2$, when $i \in I_{0}$, we conclude that, if the process starts in $I_{0}$ at time 0 , then the first time $\tau$ it will reach $\{3,4\}$ is a geometric random variable with parameter $1 / 2$ : $\operatorname{prob}(\tau=t)=1 / 2^{t}, t=1,2,3, \ldots$

As above, let $S$ denote the index of the recurrence class of $i_{\tau}$. When $i_{0}=1$, then $S=1$ whenever $\tau$ is an odd number and $S=2$ otherwise. Clearly,

$$
\operatorname{prob}\left(S=2 \mid i_{0}=1\right)=\sum_{k=1}^{\infty} \operatorname{prob}(\tau=2 k)=\sum_{k=1}^{\infty} 1 / 4^{k}=1 / 3
$$


and thus $\hat{\mu}^{\alpha, 1}=2 / 3 \mu_{1}^{\alpha}+1 / 3 \mu_{2}^{\alpha}=(0,0,2 / 3,1 / 3)$.

A similar argument shows that $\hat{\mu}^{\alpha, 2}=1 / 3 \mu_{1}^{\alpha}+2 / 3 \mu_{2}^{\alpha}=(0,0,1 / 3,2 / 3)$, since, if $i_{0}=2$, then the event $\{S=1\}$ corresponds to the even values of $\tau$.

Clearly

$$
\xi^{\alpha}=\frac{1}{36}(7,8,10,11)
$$

which yields

$$
\hat{\mu}^{\alpha}=\left(0,0, \frac{13}{27}, \frac{14}{27}\right)
$$

and hence $v_{1}^{\alpha}, v_{2}^{\alpha}, v_{3}^{\alpha}$ and $v_{4}^{\alpha}$ converge to $0,0,52 / 3$ and $56 / 3$.

Let us now deal with the general case where $\theta_{i}^{\alpha}$ are not all the same. We will analyze the situation where $\theta_{i}^{\alpha}$ is a function of a parameter $\theta$ going to 1 with the following hypotheses:

$$
\begin{array}{r}
\lim _{\theta \rightarrow 1} \theta_{i}^{\alpha}(\theta)=1, \text { for all } i \\
\theta_{i}^{\alpha}(\theta)<1, \text { for all } i \text { and } \theta<1 . \\
0<\lim _{\theta \rightarrow 1} \frac{1-\theta_{i}^{\alpha}(\theta)}{1-\theta_{1}^{\alpha}(\theta)}=\delta_{i}^{\alpha}<\infty
\end{array}
$$

For simplicity, we also will assume that $\mathcal{I}=I_{1}^{\alpha}$, i.e., there is just one recurrent class comprising all the nodes.

Theorem 4 Under (12), (13), (14), v $v_{i}^{\alpha}$ converges to $y^{\alpha} \frac{\delta_{i}^{\alpha} \mu_{i}^{\alpha}}{\sum_{j \in \mathcal{I}} \delta_{j}^{\alpha} \mu_{j}^{\alpha}}$ as $\theta$ tends to 1.

\subsection{Impact of the Social Network on Nash Equilibria}

As one might expect, the strategic behavior of firms in NE is heavily affected by the underlying social network which, after all, determines the "game form" of the competition. We already saw glimpses of this in Section 3.3 on regionalized vs unified customer territories; and in Theorem 3 and its Corollary in Section 3.4, where the money spent on a costumer was shown to be linked to his connectivity in the social network. (Look ahead also at Theorem 9 on nested clientele in Section 4.6).

Here we describe two other ways in which the network can have impact ${ }^{9}$ on NE. Note that the uniqueness of NE is a fortunate feature of our model and it makes the analysis that much more tractable.

First, consider the case when the game is anonymous (Section 3.3). Suppose the influence that customer $j$ wields on one of his neighbors in the social network is increased. In other words, suppose that $w_{i j}$ goes up for some $i$, while the rest of the matrix $W$ stays fixed. We show that this causes more money to be spent on $j$ by all the firms, not just in absolute, but also in relative, terms. Precisely we have:

Theorem 5 Assume that the game is anonymous as in Section 3.3. Suppose the social network is changed from $W$ to $\tilde{W}$, with $\tilde{w}_{i j}>w_{i j}$ (without violating the sub-stochasticity of row $i$ ) and $\tilde{w}_{k l}=w_{k l}$ for all $(k, l) \neq(i, j)$. Suppose also that $u \gg 0$. Let $m^{\alpha}, \tilde{m}^{\alpha}$ denote the money spent by firm $\alpha$ on customers in the unique NE under $W, \tilde{W}$ respectively. Then the set of blockaded and active firms is the same in both NE. For all active firms $\alpha$, we have $\tilde{m}_{j}^{\alpha}>m_{j}^{\alpha}$; furthermore,

$$
\frac{\tilde{m}_{j}^{\alpha}}{\tilde{m}_{k}^{\alpha}} \geq \frac{m_{j}^{\alpha}}{m_{k}^{\alpha}}
$$

for all $k \in \mathcal{I}$.

\footnotetext{
${ }^{9}$ This is a promising area for further exploration, and our results are indicative of the kind of structure one may hope to uncover.
} 
Remark: The above Theorem, applied iteratively, also shows that if the influence of $j$ on several of his neighbors is enhanced, the conclusion of the Theorem continues to hold.

The situation is more subtle in the non-anonymous case when firms have different characteristics $u^{\alpha}, c^{\alpha}, W^{\alpha}, \Theta^{\alpha}$. We leave it for future research to explore conditions under which analogous results will hold.

Next consider the scenario where externalities become dominant as in Section 3.4. Suppose, in the social network for firm $\alpha$, that there is a customer $j$ who influences others but himself remains uninfluenced (rather like the characters in Shakespeare's 94th sonnet ${ }^{10}$ ). Then $j$ by himself will form a recurrent class in the Markov chain associated with the network. It follows from our analysis that firm $\alpha^{\prime} s$ expenditures will be 0 on the customers that are in $j / s$ sphere of influence ${ }^{11}$, and will instead be bestowed on $j$. Of course there may be other Shakespearean characters (opinion-making leaders) akin to $j$ in firm $\alpha^{\prime} s$ network. They could all vie with each other for $\alpha^{\prime} s$ money. Much of firm $\alpha^{\prime} s$ money will be bestowed collectively on them (indeed all of it will be so bestowed, unless there is also a pool of customers in the network who mutually influence each other and form a separate recurrent class).

\section{Model with Bounded Expenditures}

\subsection{Bounded Expenditures}

The quasi-linear model can easily be extended to incorporate continuous, convex, nondecreasing functions $C^{\alpha}$ defined either on a compact, convex subset of $R_{+}^{\mathcal{I}}$ or on the whole space provided $C^{\alpha}\left(m^{\alpha}\right) \rightarrow \infty$ as $\left\|m^{\alpha}\right\| \rightarrow \infty$.

NE continue to exist under assumptions AI and AII of Theorem 1. (This follows from Theorem 10 in section 5.)

In particular, we shall focus on the fixed-budget case where each firm $\alpha$ can, at no cost, spend a total of $M^{\alpha}$ in any way across the costumers.

\subsection{Multiple Nash Equilibria}

Unfortunately it is no longer true that NE are unique. Consider the following simple fixed-budget example. There are two customers and two firms. The budgets of the two firms are identical: $M^{1}=M^{2}=1$. Suppose $v_{1}^{2}=v_{1}^{1}=1, v_{2}^{2}=0.02$ and $v_{2}^{1}=500$. (The $u_{i}^{\alpha}$ can be adjusted, given any $w_{i j}^{\alpha}>0$ and $0 \leq \theta^{\alpha}<1$, to guarantee that the $v_{i}^{\alpha}$ take on these values.) Finally take our canonical marketing function, i.e., $\gamma_{i}^{\alpha}(m)=m_{i}^{\alpha} / \bar{m}_{i}$ if $\bar{m}_{i}>0$ and 0 otherwise. By Theorem 10 there exists an NE; and, if $m$ is any NE, we must have $\bar{m}_{1}>0$ and $\bar{m}_{2}>0$. Now if $m_{i}^{\alpha}=0$ for any $\alpha$ and $i$ then the rival firm $\beta$ can reduce $m_{i}^{\beta}$ and shift money to the other client $j \neq i$, improving its payoff. We conclude that $m_{i}^{\alpha}>0$ for $i=1,2$ and $\alpha=1,2$. Therefore $m$ is an NE if, and only if,

\footnotetext{
$10 " . .$. Who, moving others, are themselves as stone

Unmoved, cold, and to temptation slow ...

They are the lords and owners of their faces

Others but stewards of their excellence......."

${ }^{11}$ Drawing a directed arc from $i$ to $j$ if $w_{i j}^{\alpha}>0$ (i.e., if $j$ influences $i$ in $\alpha^{\prime} s$ social network), the "sphere of influence" of $j$ consists of all $k$ such that there a directed path from $k$ to $j$ (allowing $j$ to influence $k$ through a chain of intermediaries)
} 
the first order conditions below hold

$$
\begin{aligned}
& \frac{v_{1}^{1} m_{1}^{2}}{\left(m_{1}^{1}+m_{1}^{2}\right)^{2}}=\frac{v_{2}^{1} m_{2}^{2}}{\left(m_{2}^{1}+m_{2}^{2}\right)^{2}}:=c^{1}, \\
& \frac{v_{1}^{2} m_{1}^{1}}{\left(m_{1}^{1}+m_{1}^{2}\right)^{2}}=\frac{v_{2}^{2} m_{2}^{1}}{\left(m_{2}^{1}+m_{2}^{2}\right)^{2}}:=c^{2}
\end{aligned}
$$

along with $m>>0, m_{1}^{1}+m_{2}^{1}=M^{1}$ and $m_{1}^{2}+m_{2}^{2}=M^{2}$. (Here $c^{\alpha}$ can be interpreted as the marginal utility of a dollar for company $\alpha$ at the NE).

By straightforward algebra, we obtain

$$
\begin{array}{r}
m_{i}^{1}=\frac{c_{2}\left(v_{i}^{1}\right)^{2} v_{i}^{2}}{\left(c_{1} v_{i}^{2}+c_{2} v_{i}^{1}\right)^{2}}, \\
m_{i}^{2}=\frac{c_{1}\left(v_{i}^{2}\right)^{2} v_{i}^{1}}{\left(c_{1} v_{i}^{2}+c_{2} v_{i}^{1}\right)^{2}}, \\
M^{1}+M^{2}=\sum_{i=1}^{2} v_{i}^{1} v_{i}^{2} \frac{1}{\left(c_{1} v_{i}^{2}+c_{2} v_{i}^{1}\right)}
\end{array}
$$

and

$$
\sum_{i=1}^{2} \frac{v_{i}^{1} v_{i}^{2}}{\left(c_{1} v_{i}^{2}+c_{2} v_{i}^{1}\right)^{2}}\left(M^{2} c_{2} v_{i}^{1}-M^{1} c_{1} v_{i}^{2}\right)=0
$$

Clearly if $\left(c_{1}, c_{2}\right)$ solves $(20)$, then so does $\left(\lambda c_{1}, \lambda c_{2}\right)$ for any $\lambda>0$. So consider (20) with $c_{2}=1$, which yields (substituting our values for $v_{i}^{\alpha}$ and $M^{\alpha}$ ) a cubic equation in $c_{1}$ with three positive roots, whose approximate values are $\tilde{c}_{1}=1.087109, \tilde{c_{1}}=47.1973$, and $\tilde{c_{1}}=24800.020967164826$. But in order to satisfy (19) we must have $c_{2}=\lambda, c_{1}=\lambda \tilde{c_{1}}$, where $\lambda$ satisfies

$$
\lambda=\frac{1}{2}\left(\frac{1}{1+\tilde{c}_{1}}+\frac{10}{500+0.02 \tilde{c}_{1}}\right)
$$

Thus we get three distinct pairs $\left(c_{1}, c_{2}\right) \approx(0.271305,0.249565),\left(c_{1}, c_{2}\right) \approx(0.96071,0.0203552)$ and $\left(c_{1}, c_{2}\right) \approx$ $(125,0.0050402)$ which give (via (17) and (18)) three distinct $m, \tilde{m}$ and $\hat{m}$ as NE:

$$
\begin{aligned}
& m_{1}^{1} \approx 0.919868, \quad m_{2}^{1}=1-m_{1}^{1} \\
& m_{1}^{2} \approx 0.999996, \quad m_{2}^{2}=1-m_{1}^{2}
\end{aligned}
$$

and

$$
\begin{gathered}
\tilde{m}_{1}^{1} \approx 0.0211485, \quad \tilde{m}_{2}^{1}=1-\tilde{m}_{1}^{1} \\
\tilde{m}_{1}^{2} \approx 0.998152, \quad \tilde{m}_{2}^{2}=1-\tilde{m}_{1}^{2}
\end{gathered}
$$

and

$$
\begin{gathered}
\hat{m}_{1}^{1} \approx 3.2256 \times 10^{-7}, \quad \hat{m}_{2}^{1}=1-\hat{m}_{1}^{1} \\
\hat{m}_{1}^{2} \approx 0.0079995, \quad \hat{m}_{2}^{2}=1-\hat{m}_{1}^{2}
\end{gathered}
$$

(The reader may numerically check that $m, \tilde{m}$ and $\hat{m}$ are indeed approximate solutions to (15) and (16).)

Notice that the two companies have widely disparate valuations of client 2 in our counter example: $v_{2}^{1}=500$ and $v_{2}^{2}=0.02$. Curiously, if we replicate each company, the counterexample disappears and uniqueness of $\mathrm{NE}$ is 
restored. More generally uniqueness holds if, for each company there are "sufficiently many" other companies whose characteristics are "nearby" ${ }^{2}$. Of course the words in quotes must be made precise (which we shall do in Section 4.4).

But the example does show the need to impose additional constraints on the marketing functions $\gamma_{i}^{\alpha}$ to guarantee uniqueness of $\mathrm{NE}$, a matter to which we now turn.

\subsection{Uniqueness with Multi-Concavity}

We shall first present an abstract result and later bring it to bear on our model. Let $S^{\alpha} \subset R_{+}^{\mathcal{I}}$ be the (closed, convex) strategy-set of $\alpha \in \mathcal{A}$. Given a strategy profile $\left(m^{\beta}\right)_{\beta \in \mathcal{A}} \in \times_{\beta \in \mathcal{A}} S^{\beta}$, suppose the payoff to any firm $\alpha$ depends on his action $m^{\alpha}$ and the aggregate $\sum_{\beta \in \mathcal{A} \backslash\{\alpha\}} m^{\beta}$ of others' actions. So we may take $\alpha$ 's payoff $\Pi^{\alpha}$ to be defined on $S^{\alpha} \times R_{+}^{\mathcal{I}}$. It will be convenient to extend the domain of $\Pi^{\alpha}$ to $R^{\mathcal{I}} \times R_{+}^{\mathcal{I}}$ by putting $\Pi^{\alpha}\left(m^{\alpha},.\right)=-\infty$ if $m^{\alpha} \notin S^{\alpha}$. Assume $\Pi^{\alpha}\left(m_{i}^{\alpha}, m_{-i}^{-\alpha}\right)$ is concave in $m_{i}^{\alpha}$ for any fixed $m_{-i}^{-\alpha}$, and let $\partial_{m_{i}^{\alpha}} \Pi^{\alpha}(m)$ denote its super-differential w.r.t. $m_{i}^{\alpha}$.

Theorem 6 Denote $\bar{m}^{\alpha}=\sum_{i \in \mathcal{I}} m_{i}^{\alpha}$. Suppose the following conditions hold on a set $\mathcal{N} \subset \prod_{\beta \in \mathcal{A}} S^{\beta}$ : for all $\alpha$ and $i$, the super-differential $\partial_{m_{i}^{\alpha}} \Pi^{\alpha}(m)$ is a correspondence $h_{i}^{\alpha}\left(m_{i}^{\alpha}, \bar{m}_{i}, \bar{m}^{\alpha}\right)$ that is strictly decreasing ${ }^{13}$ in $m_{i}^{\alpha}$ and non-increasing in both $\bar{m}_{i}$ and $\bar{m}^{\alpha}$. Then there is at most one $N E$ in $\mathcal{N}$.

To apply Theorem 6 to our model, we focus on the case when costs are convex in total expenditure and benefits are linear, so that the payoff function may be written

$$
\Pi^{\alpha}(m)=\sum_{i \in \mathcal{I}} v_{i}^{\alpha} \gamma_{i}^{\alpha}\left(m_{i}\right)-C^{\alpha}\left(\bar{m}^{\alpha}\right)
$$

We shall refer to this in brief as "the quasi-linear model with convex costs". Note that we are not insisting that the function $C^{\alpha}$ be continuous. Define the function $C^{\alpha}$ by: $C^{\alpha}\left(m^{\alpha}\right)=0$ if $m^{\alpha} \in S^{\alpha}$ and $C^{\alpha}\left(m^{\alpha}\right)=\infty$ otherwise. Note that $C^{\alpha}$ is convex if $S^{\alpha}$ is convex. Hence the fixed budget case is included in this model.

The super-differential of $-C^{\alpha}$ is clearly decreasing in $\bar{m}^{\alpha}$ since $C^{\alpha}$ is convex. It therefore suffices to check that the super-differential of $\gamma_{i}^{\alpha}$ can be expressed as a correspondence of two variables $m_{i}^{\alpha}$ and $\bar{m}_{i}$, which is strictly decreasing in $m_{i}^{\alpha}$ and decreasing in $\bar{m}_{i}$.

Consider, for concreteness, our canonical marketing function $\gamma_{i}^{\alpha}=m_{i}^{\alpha} / \bar{m}_{i}$. Note

$$
\frac{\partial \gamma_{i}^{\alpha}}{\partial m_{i}^{\alpha}}=\frac{\bar{m}_{i}-m_{i}^{\alpha}}{\left(\bar{m}_{i}\right)^{2}}:=h\left(m_{i}^{\alpha}, \bar{m}_{i}\right)
$$

Clearly $h$ is strictly decreasing in $m_{i}^{\alpha}$. But

$$
\frac{\partial h}{\partial \bar{m}_{i}}=\frac{-\bar{m}_{i}+2 m_{i}^{\alpha}}{\left(\bar{m}_{i}\right)^{3}}
$$

is non-positive if, and only if, $m_{i}^{\alpha} \leq \bar{m}_{i} / 2$.

In the light of this, Theorem 6 immediately yields

\footnotetext{
${ }^{12}$ As we expand the neighborhood of characteristics that defines "nearby", we will need to put in more companies in that neighborhood.

${ }^{13} \mathrm{~A}$ correspondence $\Lambda(x, y, z)$ is said to be non-increasing in $x$ on a set $\mathcal{V}$ if$$
\left\{v \in \Lambda(x, y, z), w \in \Lambda\left(x^{\prime}, y, z\right), x^{\prime}>x,(x, y, z) \in \mathcal{V},\left(x^{\prime}, y, z\right) \in \mathcal{V}\right\} \Rightarrow v \geq w .
$$

If the last inequality is strict, we say that $\Lambda$ is strictly decreasing in $x$. 
Corollary 2 In the quasi-linear model with convex costs and canonical marketing, there is at most one NE in the region

$$
\Omega:=\left\{m: m_{i}^{\alpha} \leq \bar{m}_{i} / 2 \text { for all } \alpha \in \mathcal{A} \text { and } i \in \mathcal{I}\right\}
$$

No wonder that, in the counterexample of Section 4.2, the NE were not contained in $\Omega$.

\subsection{Competition Restores Uniqueness}

Throughout this section we confine ourselves to the fixed-budget model with the canonical marketing function, which was also the context of the counterexample.

We shall show that, with "enough competition" no NE can be outside $\Omega$. This will guarantee uniqueness of NE (by Corollary 2).

First consider the time-honored device of creating competition by replicating the companies, i.e., for any $\alpha$, there is a replica (twin) $\tilde{\alpha}$ with identical characteristics $\left(\theta^{\alpha}=\theta^{\tilde{\alpha}}, u^{\alpha}=u^{\tilde{\alpha}}, W^{\alpha}=W^{\tilde{\alpha}}, M^{\alpha}=M^{\tilde{\alpha}}\right)$. It suffices to show that replicas act identically in any NE, for then obviously $m \in \Omega$.

We shall prove this by contradiction. Suppose $m$ is an NE with $m^{\alpha} \neq m^{\tilde{\alpha}}$. Since ${ }^{14} \sum_{i \in \mathcal{I}} m_{i}^{\alpha}=M^{\alpha}=$ $M^{\tilde{\alpha}}=\sum_{i \in \mathcal{I}} m_{i}^{\tilde{\alpha}}$, there exist clients $i$ and $j$ such that $r_{i}^{\alpha}>r_{i}^{\tilde{\alpha}}$ and $r_{j}^{\alpha}<r_{j}^{\tilde{\alpha}}$ (where, recall, $r_{i}^{\alpha}:=m_{i}^{\alpha} / \bar{m}_{i}$ etc.). The first order conditions of NE are

$$
\begin{aligned}
\frac{v_{i}^{\alpha}\left(1-r_{i}^{\alpha}\right)}{\bar{m}_{i}} & \geq \frac{v_{j}^{\alpha}\left(1-r_{j}^{\alpha}\right)}{\bar{m}_{j}} \\
\frac{v_{i}^{\tilde{\alpha}}\left(1-r_{i}^{\tilde{\alpha}}\right)}{\bar{m}_{i}} & \leq \frac{v_{j}^{\tilde{\alpha}}\left(1-r_{j}^{\tilde{\alpha}}\right)}{\bar{m}_{j}}
\end{aligned}
$$

(Since $r_{i}^{\alpha}>0$, we have $m_{i}^{\alpha}>0$ and so the LHS of (21) must equal $c^{\alpha}:=$ the marginal utility of a dollar to $\alpha$ at the NE. But RHS of (21) is at most $c^{\alpha}$, proving (21). A similar argument can be made for (22).)

But $v_{i}^{\alpha}=v_{i}^{\tilde{\alpha}}$ and $v_{j}^{\alpha}=v_{j}^{\tilde{\alpha}}$ by (6). So LHS of (22)> LHS of (21) $\geq$ RHS of (21) > RHS of (22), contradicting (22).

This establishes uniqueness of NE under replication.

But it is not necessary to have exact replicas. It suffices to assume that, for each company $\alpha$, there are sufficiently many rivals whose characteristics are "close enough" to those of $\alpha$. (As we relax the notion of "closeness", we will need to put in more rivals.) Precisely, we have:

Theorem 7 Consider the quasi-linear model with fixed positive budgets ${ }^{15}$ and canonical marketing. For any $\beta \in \mathcal{A}$, denote ${ }^{16} v_{\text {min }}^{\beta}=\min \left\{v_{j}^{\beta}: j \in \mathcal{I}, v_{j}^{\beta}>0\right\}$ and $v_{\text {max }}^{\beta}=\max \left\{v_{j}^{\beta}: j \in \mathcal{I}, v_{j}^{\beta}>0\right\}$. Fix an integer $n \geq 2$. Assume that, for each $\alpha \in \mathcal{A}$ there exists $\mathcal{A}^{\alpha}(n) \subset \mathcal{A} \backslash\{\alpha\}$ such that $v_{i}^{\beta}=0$ if and only if $v_{i}^{\alpha}=0$ for all $\beta \in \mathcal{A}^{\alpha}(n)$ and all $i \in \mathcal{I}$. Furthermore assume, for every $\alpha \in \mathcal{A}$, that

$$
\begin{array}{ll}
\text { (i) } & \left|\mathcal{A}^{\alpha}(n)\right| \geq n \\
\text { (ii)(a) } & \exists k_{1}>0, k_{2}>0 \text { s.t. } k_{1} k_{2}<(2 n-1) / n \text {; and } \\
\text { (b) } & k_{1}^{-1} \leq v_{i}^{\alpha} / v_{i}^{\beta} \leq k_{2} \text { for all } \beta \in \mathcal{A}^{\alpha}(n) \text { and all } i \in\left\{j \in \mathcal{I}: v_{j}^{\alpha}>0\right\} . \\
\text { (iii) } & M^{\alpha}-M^{\beta} \leq \frac{3}{16|\mathcal{I}|}\left(v_{\text {min }}^{\beta} / v_{\text {max }}^{\beta}\right) M^{\beta} \text { for all } \beta \in \mathcal{A}^{\alpha}(n)
\end{array}
$$

Then $\Gamma$ has a unique NE.

\footnotetext{
${ }^{14}$ Clearly both the companies will spend all their money at any NE, since each puts positive value on at least one customer-node. ${ }^{15}$ Leading to convex costs (see the beginning of Section 4 ).

${ }^{16}$ Recall that $\left(u_{j}^{\beta}\right)_{j \in \mathcal{I}} \neq 0$ by assumption, hence (see (6)) we have $\left(v_{j}^{\beta}\right)_{j \in \mathcal{I}} \neq 0$.
} 


\subsection{Anonymous Valuations Restore Uniqueness}

$\mathrm{NE}$ are unique even with heterogeneous convex costs and marketing functions that are more general than our canonical example, provided that companies' valuations of clients are identical: $v_{i}^{\alpha}=v_{i}$ for all $\alpha$ and $i$. This is not an unnatural assumption. It holds when $w_{i j}^{\alpha}$ and $\theta_{i}^{\alpha}$ are invariant of $\alpha$, as in our example in Section 2.2.

Suppose that costs are given by differentiable and convex functions of total expenditure: $C^{\alpha}\left(m^{\alpha}\right):=C^{\alpha}\left(\bar{m}^{\alpha}\right)$ for all $\alpha \in \mathcal{A}$. Further suppose that the marketing impact of $\alpha$ on $i$ can be factored in terms of $i$ 's expenditure $m_{i}^{\alpha}$ and the total expenditure $\bar{m}_{i}$.

BI Suppose there exist strictly decreasing functions $\lambda^{\alpha}$ and strictly increasing functions $f_{i}$ such that:

$$
\lambda_{i}^{\alpha}\left(r_{i}^{\alpha}, \bar{m}_{i}\right)=\frac{\lambda^{\alpha}\left(r_{i}^{\alpha}\right)}{f_{i}\left(\bar{m}_{i}\right)}
$$

for all $\alpha \in \mathcal{A}$ and $i \in \mathcal{I}$, whenever $\bar{m}_{i}>0$. (Recall $r_{i}^{\alpha}:=m_{i}^{\alpha} / \bar{m}_{i}$ and $\lambda_{i}^{\alpha}$ was defined in equation (8).)

Note that, in our canonical case, $\lambda_{i}^{\alpha}\left(r_{i}^{\alpha}, \bar{m}_{i}\right)=\left(1-r_{i}^{\alpha}\right) / \bar{m}_{i}$ and so (BI) is satisfied. More generally, the key examples also satisfy (BI).

Theorem 8 Suppose $v_{i}^{\alpha}=v_{i}$ for all $\alpha$ and $i$, and that assumption BI holds. Then there exists a unique NE.

\subsection{Structure of Nash Equilibrium with Convex Costs and Anonymous Valua- tions.}

It is natural to consider the case where the marketing impact is an anonymous function of expenditures, as in our canonical example.

BII The functions $\lambda_{i}^{\alpha}$ do not depend on $\alpha$; i.e. $\lambda_{i}^{\alpha}=\lambda_{i}^{\beta}:=\lambda_{i}$ for all $\alpha, \beta$ and $i$.

Also assume that $\lambda_{i}$ is concave and increasing in $m_{i}^{\alpha}$, as in Section 2. In this event, even without the factorization of BI, we can describe an interesting structural feature of NE (though we do not know if they are unique).

Recall that $\xi^{\alpha}\left(\bar{m}^{\alpha}\right)=\left(d / d \bar{m}^{\alpha}\right) C^{\alpha}\left(\bar{m}^{\alpha}\right)$.

Theorem 9 Suppose the model stated above satisfies BI and BII. Let $m$ be any NE. Denote $\mathcal{I}(\alpha)=\{i \in \mathcal{I}$ : $\left.m_{i}^{\alpha}>0\right\}$. There is an ordering $\alpha_{1} \leq \alpha_{2} \leq \ldots \leq \alpha_{n}$ of the firms such that $\mathcal{I}\left(\alpha_{1}\right) \subset \mathcal{I}\left(\alpha_{2}\right) \subset \ldots \subset \mathcal{I}\left(\alpha_{n}\right)$. In other words the clientele of active firms are always nested.

\section{The General Model: Existence of Nash Equilibrium}

Let us first recall some basic features of our quasi-linear model.

Given any fixed $m$, customer $i$ takes $m_{i}$ into account, but then interacts with others in the social network, and revises his $p_{i}^{\alpha}$ progressively. This gives rise to a natural dynamic: if, at some time $t \geq 0$, proclivities to purchase are given by $p^{\alpha}(m, t):=\left(p_{j}^{\alpha}(m, t)\right)_{j \in \mathcal{I}}$, we will have $p_{i}^{\alpha}(m, t+1)=F_{i}^{\alpha}\left(m, p^{\alpha}(m, t)\right)$ for some function $F_{i}^{\alpha}$. Clearly $p_{i}^{\alpha}(m, t+1)$ will in general not only depend upon others proclivities $\left(p_{j}^{\alpha}(m, t)\right)_{j \neq i}$ (which is the externality effect we want to capture) but also on his own $p_{i}^{\alpha}(m, t)$; for who, even when swayed by others, is not partly held back by the inertia of his past habits? 
If we want to ensure that people's opinions settle down over time, instead of fluctuating forever or diverging without bound, then we must require the dynamic process $\left\{p^{\alpha}(m, t)\right\}_{t=1}^{\infty}$ to converge as $t \rightarrow \infty$. To this end, we shall need to impose conditions on the functions $F_{i}^{\alpha}(m, \cdot)$, which portray how customer $i$ revises his proclivities of purchases from firm $\alpha$.

The most natural assumption that comes to mind, from the mathematical standpoint is that $F_{i}^{\alpha}(m, \cdot)$ be a contraction for every $m$ (see (3) of CIV below); for then it follows that this dynamic process settles very quickly (geometrically) to a steady state $p^{\alpha}(m):=\left(p_{j}^{\alpha}(m)\right)_{j \in \mathcal{I}}$, namely the unique fixed point of $F^{\alpha}(m, \cdot):=$ $\left(F_{i}^{\alpha}(m, \cdot)\right)_{i \in \mathcal{I}}$ :

$$
p_{i}^{\alpha}(m)=F_{i}^{\alpha}\left(m, p^{\alpha}(m)\right), \text { for all } i \in \mathcal{I}, \alpha \in \mathcal{A}
$$

We shall ignore in this paper the transient phase of the dynamic because if $p^{\alpha}(m, t)$ is viewed as a proclivity to purchase, then it will only be put into effect once it becomes stable. Would a customer buy a new car of a particular company when he is still in the process of revising his mind based on the feedback from his neighbors? On the other hand, if $p^{\alpha}(m, t)$ represents actual purchases that are occurring repeatedly in small quantities, then the aggregate purchase in the steady state overwhelms the small volume traded during the very short transient phase.

In either scenario a firm need only worry about the steady state behavior of customers in evaluating its payoff. It thus seems natural to suppose that the outcome engendered by a strategy profile $m$ is the unique fixed point $p^{\alpha}(m)$ of $F^{\alpha}(m, \cdot)$. This fully defines the map from $m$ to $p(m)=\left(p^{\alpha}(m)\right)_{\alpha \in \mathcal{A}}$, and thereby the strategic game $\Gamma$ between the firms.

However, at this level of abstraction, it is hard to imagine that firms can come to know the functions $\left(F^{\alpha}\right)_{\alpha \in \mathcal{A}}$. The social interaction between customers tends to be quite subtle and it is not easy for firms to generally predict the outcome with any degree of accuracy. But there are scenarios in which the interaction gets channelled through networks that are common knowledge. In particular this is possible in the wired world where the interaction may be tracked online and made explicit. (See examples in [15] and our own example in Section 2.2 .) Then $F:=\left(F^{\alpha}\right)_{\alpha \in \mathcal{A}}$ can become "manifest" to the companies, enabling them to compute the effect of the interaction, and thus to participate in the kind of game we are describing.

Recall that even in the canonical example of our quasi-linear model (which we are endeavoring to generalize) there was no natural definition of $F_{i}^{\alpha}$ at points $m \in R_{+}^{\mathcal{I} \times \mathcal{A}}$ where $\bar{m}_{i}=0$ for some $i$. Indeed as $m$ was approached from different directions in the interior $R_{++}^{\mathcal{I} \times \mathcal{A}}$, we got different limits for $F_{i}^{\alpha}$, ruling out any possibility of a continuous extension of $F_{i}^{\alpha}$ to $m$.

We therefore start with the set of "interior" points

$$
\mathcal{M}:=\left\{m \in R_{+}^{\mathcal{I} \times \mathcal{A}}: \bar{m}_{i}>0, \forall i\right\}
$$

at which all customer markets are active. Conditions on $F$ will first be described on the domain ${ }^{17} \mathcal{M}$ (see CIV below). Later we shall impose additional constraints on $F$ at the "boundary" points $R_{+}^{\mathcal{I} \times \mathcal{A}} \backslash \mathcal{M}$, in terms of the limiting behavior of $F$ (see CV below). Needless to say both CIV and CV will be satisfied by the quasi-linear model.

Let us now describe our assumptions in the general model.

\footnotetext{
${ }^{17}$ The reader will notice that we cannot restrict $F$ to $\mathcal{M}$, as the set $\mathcal{M}$ is not a Cartesian product and thus unable to incorporate independent strategy choices of the firms. Nor can we restrict $F$ to $R_{++}^{\mathcal{I} \times \mathcal{A}}$, because we must allow for the natural possibility that some firms may want to put no money on a particular customer.
} 
CI : $\forall \alpha$, the cost function $C^{\alpha}: Z^{\alpha} \rightarrow R_{+}$is continuous, convex and nondecreasing ${ }^{18}$, where $Z^{\alpha} \subset R_{+}^{\mathcal{I}}$ is a convex neighborhood of the origin in $R_{+}^{\mathcal{I}}$. Furthermore $Z^{\alpha}$ is either compact or else $Z^{\alpha}=R_{+}^{\mathcal{I}}$; and in the latter case $C\left(m^{\alpha}\right) \rightarrow \infty$ as $\left\|m^{\alpha}\right\| \rightarrow \infty$.

CII : $\forall \alpha$, the benefit function $U^{\alpha}:[0,1]^{\mathcal{I}} \rightarrow R$ is continuous, concave and nondecreasing.

Before stating the other assumptions, we shall need some definitions. For $\alpha \in \mathcal{A}$, we define the partial order $\underbrace{\alpha}$ on $[0,1]^{\mathcal{I} \times \mathcal{A}}$ by:

$$
x \stackrel{\alpha}{\succeq} y \text { if and only if } \forall i \in \mathcal{I}, x_{i}^{\alpha} \geq y_{i}^{\alpha} \text { and } \forall \beta \neq \alpha, \forall i \in \mathcal{I}, x_{i}^{\beta} \leq y_{i}^{\beta}
$$

We extend the order $\stackrel{\alpha}{\succeq}$ to $R^{\mathcal{I}} \times[0,1]^{\mathcal{I} \times \mathcal{A}}$ by the rule

$$
\left(\hat{m}^{\alpha}, \hat{p}\right) \stackrel{\alpha}{\succeq}\left(m^{\alpha}, p\right) \text { if } \hat{m}^{\alpha} \geq m^{\alpha} \text { and } \hat{p} \stackrel{\alpha}{\succeq} p
$$

We say that a function $H: R^{\mathcal{I}} \times[0,1]^{\mathcal{I} \times \mathcal{A}} \rightarrow[0,1]^{\mathcal{I} \times \mathcal{A}}\left(\right.$ resp. $\left.H: R^{\mathcal{I}} \times[0,1]^{\mathcal{I} \times \mathcal{A}} \rightarrow R^{\mathcal{I}} \times[0,1]^{\mathcal{I} \times \mathcal{A}}\right)$ is $\alpha$-nondecreasing if $\left(\tilde{m}^{\alpha}, \tilde{p}\right) \stackrel{\alpha}{\succeq}\left(m^{\alpha}, p\right)$ implies $H\left(\tilde{m}^{\alpha}, \tilde{p}\right) \succeq H\left(m^{\alpha}, p\right)$.

The function $H$ is said to be $\alpha$-concave if $\left(m_{\lambda}^{\alpha}, p_{\lambda}\right):=\lambda\left(m^{\alpha}, p\right)+(1-\lambda)\left(\tilde{m}^{\alpha}, \tilde{p}\right)$, with $\lambda \in[0,1]$, implies $H\left(m_{\lambda}^{\alpha}, p_{\lambda}\right) \stackrel{\alpha}{\succeq} \lambda H\left(m^{\alpha}, p\right)+(1-\lambda) H\left(\tilde{m}^{\alpha}, \tilde{p}\right)$.

We are ready to state our next assumption.

CIII (1) $\forall \alpha, \forall p, \forall m=\left(m^{-\alpha}, m^{\alpha}\right) \in R_{+}^{\mathcal{I} \times \mathcal{A}}: F\left(m^{-\alpha}, m^{\alpha}, p\right)$ is $\alpha$-nondecreasing in $\left(m^{\alpha}, p\right)$ at fixed $m^{-\alpha}$.

(2) For all $m \in \mathcal{M}, \forall \alpha, F\left(m^{-\alpha}, m^{\alpha}, p\right)$ is continuous in all its variables; furthermore, it is $\alpha$-concave in the pair $\left(m^{\alpha}, p\right)$ for any fixed $m^{-\alpha}$.

(3) $F(m, p)$ is a contraction in $p$, uniformly in $m \in R_{+}^{\mathcal{I} \times \mathcal{A}}$. i.e. there exists $K<1$ such that

$$
\|F(m, p)-F(m, \tilde{p})\| \leq K\|p-\tilde{p}\|, \text { for all } m, p, \tilde{p}
$$

where $\|\cdot\|$ denotes the maximum norm.

Finally we state assumptions CIV and CV near, or at, boundary points $m$ where some $\bar{m}_{i}=0$. These are just reformulations of AI and AII in the context of our general model, and have the same intuitive motivation as before.

CIV For each customer $i$, there is a set $\mathcal{A}_{i}$ of at least two firms satisfying:

(1) Every firm $\alpha$ in $\mathcal{A}_{i}$ values customer $i$, i.e., $U^{\alpha}$ is strictly increasing in the variable $p_{i}^{\alpha}$.

(2) $\forall i \in \mathcal{I}, \forall \alpha \in \mathcal{A}_{i}$, for all sequences $\left\{m_{k}\right\}_{k \in N} \subset \mathcal{M}$ such that $m_{k, i}^{\alpha} \leq \bar{m}_{k, i} / 2$ for all $k$ and $\bar{m}_{k, i} \rightarrow 0$ as $k \rightarrow \infty$, the following holds:

$$
\lim _{\delta \rightarrow 0}\left\{\limsup _{k \rightarrow \infty}\left\{\inf _{p \in[0,1]}\left[F_{i}^{\alpha}\left(m_{k}+\delta e_{i}^{\alpha}, p\right)-F_{i}^{\alpha}\left(m_{k}, p\right)\right] / \delta\right\}\right\}=\infty
$$

CV For all $m \in \mathcal{M}^{c}:=R_{+}^{\mathcal{I} \times \mathcal{A}} \backslash \mathcal{M}$, we require that $\forall i$ such that $\bar{m}_{i}=0, \forall \alpha \in \mathcal{A}_{i}$,

$$
\lim _{\epsilon \rightarrow 0} \inf _{p}\left[F_{i}^{\alpha}\left(m+\epsilon e_{i}^{\alpha}, p\right)-F_{i}^{\alpha}(m, p)\right] / \epsilon=\infty
$$

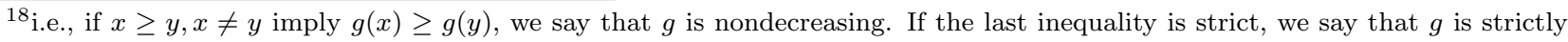
increasing.
} 
We are ready to state our main existence result.

Theorem 10 Assume $C I, C I I, C I I I, C I V$. Then an NE exists in $\mathcal{M}$ for the game $\Gamma$.

Moreover, if $C V$ is satisfied, then every $N E$ of $\Gamma$ belongs to $\mathcal{M}$.

Remark: If $F_{i}^{\alpha}$ is continuous in $m$ (even when $m \rightarrow 0$ ) then CIV can be dropped. It need only be postulated for those $i$ where continuity fails. Existence of NE remains intact, but now the total money spent on a client may be zero.

\subsection{Cross Effects}

We show that cross-effects (of $p_{j}^{\beta}$ on $p_{i}^{\alpha}$ ) can be incorporated, under some constraints, in our model without endangering the existence of NE.

For $\alpha \in \mathcal{A}$, define the partial order $\succeq^{\alpha}$ on $R^{\mathcal{I} \times \mathcal{A}}$ by:

$$
x \stackrel{\alpha}{\succeq} y \text { if and only if } \forall i \in \mathcal{I}, x_{i}^{\alpha} \geq y_{i}^{\alpha} \text { and } \forall \beta \neq \alpha, \forall i \in \mathcal{I}, x_{i}^{\beta} \leq y_{i}^{\beta}
$$

Assume that the contraction mapping $F(m, p)$ can be written as $F(m, p)=(1-\theta) \gamma(m)+\theta G(p)$ where $G$ is non-expansive (this includes our quasi-linear model). Our hypotheses on $\gamma, G$ are (assuming throughout that $m \in \mathcal{M}):$

CVI $\gamma_{i}^{\alpha}$ is concave in $m_{i}^{\alpha}$, fixing $m_{-i}^{-\alpha}$; and is convex in $m_{i}^{\beta}$, for $\beta \in \mathcal{A} \backslash\{\alpha\}$, fixing $m_{-i}^{-\beta}$.

CVII $G$ is affine and $\alpha$-increasing (i.e., $p \stackrel{\alpha}{\succeq} p^{\prime}$ implies $\left.G(p) \stackrel{\alpha}{\succeq} G\left(p^{\prime}\right)\right)$.

(It can easily be checked that our canonical example satisfies CVI .)

Theorem 11 If CI till CVII hold in the above model with cross effects, then an NE always exists.

It is worth noting that the key property invoked for Theorem 11 is that $G$ be affine and $\alpha$-increasing. Thus writing $p_{i}^{\alpha}=k_{i}^{\alpha}+\sum_{\beta \in \mathcal{A}, j \in \mathcal{I} \backslash\{i\}} w_{i j}^{\alpha \beta} p_{j}^{\beta}$ we must have

$$
\begin{aligned}
& w_{i j}^{\alpha \beta} \geq 0 \text { if } \beta=\alpha \\
& w_{i j}^{\alpha \beta} \leq 0 \text { if } \beta \neq \alpha
\end{aligned}
$$

Of course additional constraints need to be imposed on the $w_{i j}^{\alpha \beta}$ to ensure that $G$ is non-expansive (e.g., $0 \leq \sum_{\beta \in \mathcal{A}, j \in \mathcal{I} \backslash\{i\}} w_{i j}^{\alpha \beta} \leq 1$ will suffice).

\section{Appendix}

We shall begin with the proof of Theorem 10 since it implies the existence of NE for our quasi-linear model. 


\subsection{Proof of Theorem 10}

We shall first prove the Theorem when $Z^{\alpha}=R_{+}^{\mathcal{I} \times \mathcal{A}}$ and later describe the amendments needed when $Z^{\alpha}$ is compact.

For $\alpha \in \mathcal{A}$, define the partial order $\stackrel{\alpha}{\succeq}$ on $[0,1]^{\mathcal{I} \times \mathcal{A}}$ by:

$$
x \stackrel{\alpha}{\succeq} y \text { if and only if } \forall i \in \mathcal{I}, x_{i}^{\alpha} \geq y_{i}^{\alpha} \text { and } \forall \beta \neq \alpha, \forall i \in \mathcal{I}, x_{i}^{\beta} \leq y_{i}^{\beta}
$$

We extend the order $\stackrel{\alpha}{\succeq}$ to $R^{\mathcal{I}} \times[0,1]^{\mathcal{I} \times \mathcal{A}}$ by the rule

$$
\left(\hat{m}^{\alpha}, \hat{p}\right) \stackrel{\alpha}{\succeq}\left(m^{\alpha}, p\right) \text { if } \hat{m}^{\alpha} \geq m^{\alpha} \text { and } \hat{p} \stackrel{\alpha}{\succeq} p
$$

Step 1: We start with a definition. A function $G: R_{+}^{\mathcal{I} \times \mathcal{A}} \times[0,1]^{\mathcal{I} \times \mathcal{A}} \rightarrow[0,1]^{\mathcal{I} \times \mathcal{A}}$ is said to have property $(P)$ if the following requirements are met:

(P1) $\forall \alpha, \forall p, \forall m=\left(m^{-\alpha}, m^{\alpha}\right) \in R_{+}^{\mathcal{I} \times \mathcal{A}}: G\left(m^{-\alpha}, m^{\alpha}, p\right)$ is $\alpha$-nondecreasing in $\left(m^{\alpha}, p\right)$ at fixed $m^{-\alpha}$.

(P2) $\forall \alpha, \forall m=\left(m^{-\alpha}, m^{\alpha}\right) \in \mathcal{M}, \forall p: G\left(m^{-\alpha}, m^{\alpha}, p\right)$ is continuous in all its variables; furthermore, it is $\alpha$-concave in the pair $\left(m^{\alpha}, p\right)$ for any fixed $m^{-\alpha}$.

(P3) $\forall i \in \mathcal{I}, \forall \alpha, \forall p, \forall m$ and $\hat{m}$ in $R_{+}^{\mathcal{I} \times \mathcal{A}}$ with $m^{-\alpha}=\hat{m}^{-\alpha}$ and $m^{\alpha} \leq \hat{m}^{\alpha}$,

$$
G_{i}^{\alpha}(\hat{m}, p)-G_{i}^{\alpha}(m, p) \geq \inf _{\hat{p}}\left[F_{i}^{\alpha}(\hat{m}, \hat{p})-F_{i}^{\alpha}(m, \hat{p})\right]
$$

We then have the following Lemma:

Lemma 1 Let us define inductively the following sequence $\left(G_{n}\right)_{n \in N}$ of functions :

$G_{1}=F$ and, for $n>1, G_{n}(m, p):=F\left(m, G_{n-1}(m, p)\right)$. Then, for all $n, G_{n}$ has the property $(P)$.

Proof of Lemma 1: First observe that $G_{1}=F$ obviously satisfies (P) on account of CIII and CIV .

Assume inductively that $G_{n-1}$ satisfies property (P). Then, at fixed $m^{-\alpha}$, the map

$$
\chi:\left(m^{\alpha}, p\right) \rightarrow\left(m^{\alpha}, G_{n-1}\left(m^{-\alpha}, m^{\alpha}, p^{\alpha}\right)\right)
$$

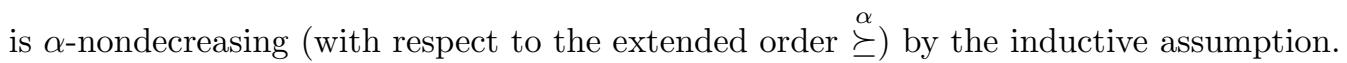

So, if $\left(\tilde{m}^{\alpha}, \tilde{p}\right) \stackrel{\alpha}{\succeq}\left(m^{\alpha}, p\right)$, then $\chi\left(\tilde{m}^{\alpha}, \tilde{p}\right) \stackrel{\alpha}{\succeq} \chi\left(m^{\alpha}, p\right)$; and since $F_{i}^{\alpha}\left(m^{-\alpha}, m^{\alpha}, p^{\alpha}\right)$ is $\alpha$-nondecreasing in $\left(m^{\alpha}, p^{\alpha}\right)$, it follows that $G_{n}\left(m^{-\alpha}, m^{\alpha}, p\right)=F\left(m^{-\alpha}, \chi\left(m^{\alpha}, p\right)\right) \stackrel{\alpha}{\preceq} F\left(m^{-\alpha}, \chi\left(\tilde{m}^{\alpha}, \tilde{p}\right)\right)=G_{n}\left(m^{-\alpha}, \tilde{m}^{\alpha}, \tilde{p}\right)$. This proves (P1) for $G_{n}$.

Continuity of $G_{n}$ follows from the fact that it is a composition of continuous functions. Next we check concavity. By the inductive assumption the map $\chi$ is $\alpha$-concave $($ since $m \in \mathcal{M})$. Let $\left(m_{\lambda}^{\alpha}, p_{\lambda}^{\alpha}\right)=\lambda\left(m^{\alpha}, p^{\alpha}\right)+$ $(1-\lambda)\left(\hat{m}^{\alpha}, \hat{p}^{\alpha}\right)$, with $\lambda \in[0,1]$. Then

$$
\chi\left(m_{\lambda}^{\alpha}, p_{\lambda}^{\alpha}\right) \stackrel{\alpha}{\succeq} \lambda \chi\left(m^{\alpha}, p^{\alpha}\right)+(1-\lambda) \chi\left(m^{\alpha}, \hat{p}^{\alpha}\right)
$$

Now,

$$
\begin{aligned}
G_{n}\left(m^{-\alpha}, m_{\lambda}^{\alpha}, p_{\lambda}^{\alpha}\right) & =F\left(m^{-\alpha}, \chi\left(m_{\lambda}^{\alpha}, p_{\lambda}^{\alpha}\right)\right) \\
& \succeq F\left(m^{-\alpha}, \lambda \chi\left(m^{\alpha}, p^{\alpha}\right)+(1-\lambda) \chi\left(m^{\alpha}, \hat{p}^{\alpha}\right)\right) \\
& \succeq \lambda F\left(m^{-\alpha}, \chi\left(m^{\alpha}, p^{\alpha}\right)\right)+(1-\lambda) F\left(m^{-\alpha}, \chi\left(m^{\alpha}, \hat{p}^{\alpha}\right)\right) \\
& =\lambda G_{n}\left(m^{-\alpha}, m^{\alpha}, p^{\alpha}\right)+(1-\lambda) G_{n}\left(m^{-\alpha}, m^{\alpha}, \hat{p}^{\alpha}\right)
\end{aligned}
$$


where the first inequality comes from $(24)$ and the fact that $F\left(m^{-\alpha}, m^{\alpha}, p^{\alpha}\right)$ is $\alpha$-nondecreasing in $\left(m^{\alpha}, p^{\alpha}\right)$; and the second inequality follows from the fact that $F\left(m^{-\alpha}, m^{\alpha}, p^{\alpha}\right)$ is $\alpha$-concave in $\left(m^{\alpha}, p^{\alpha}\right)$. This proves (P2) for $G_{n}$.

We turn to (P3). For all $i \in \mathcal{I}, \forall \alpha, \forall p, \forall m$ and $\hat{m}$ in $R_{+}^{\mathcal{I} \times \mathcal{A}}$ with $m^{-\alpha}=\hat{m}^{-\alpha}$ and $m^{\alpha} \leq \hat{m}^{\alpha}$,

$$
\begin{aligned}
{\left[G_{n, i}^{\alpha}(\hat{m}, p)-G_{n, i}^{\alpha}(m, p)\right]=} & {\left[F_{i}^{\alpha}\left(\hat{m}, G_{n-1}(\hat{m}, p)\right)-F_{i}^{\alpha}\left(m, G_{n-1}(m, p)\right)\right] } \\
= & {\left[F_{i}^{\alpha}\left(\hat{m}, G_{n-1}(\hat{m}, p)\right)-F_{i}^{\alpha}\left(m, G_{n-1}(\hat{m}, p)\right)\right] } \\
& +\left[F_{i}^{\alpha}\left(m, G_{n-1}(\hat{m}, p)\right)-F_{i}^{\alpha}\left(m, G_{n-1}(m, p)\right)\right]
\end{aligned}
$$

The first summand is bounded from below by $\inf _{q}\left[F_{i}^{\alpha}(\hat{m}, q)-F_{i}^{\alpha}(m, q)\right]$; and the second is nonnegative since $G_{n-1}$ is $\alpha$-nondecreasing in $\left(m^{\alpha}, p\right)$ for fixed $m^{-\alpha}$ by $(\mathrm{P} 1)$ and since $F$ is $\alpha$-nondecreasing in $\left(m^{\alpha}, p\right)$ by $(1)$ of CIII . This proves (P3) for $G_{n}$.

\section{Corollary 3}

(1) For all $i \in \mathcal{I}$ and $\alpha \in \mathcal{A}$,

a) $\forall m \in R_{+}^{\mathcal{I} \times \mathcal{A}}, p_{i}^{\alpha}(m)=p_{i}^{\alpha}\left(m^{\alpha}, m^{-\alpha}\right)$ is nondecreasing in $m^{\alpha}$ for every fixed $m^{-\alpha}$.

b) $\forall m \in \mathcal{M}, p_{i}^{\alpha}(m)=p_{i}^{\alpha}\left(m^{\alpha}, m^{-\alpha}\right)$ is continuous in $m$ and concave in $m^{\alpha}$ for every fixed $m^{-\alpha}$.

(2) $\forall i \in \mathcal{I}, \forall \alpha \in \mathcal{A}_{i}$, for all sequence $\left\{m_{k}\right\}_{k \in N} \subset \mathcal{M}$ such that $m_{k, i} \rightarrow 0$, and $m_{k, i}^{\alpha} \leq \bar{m}_{k, i} / 2$,

$$
\lim _{\delta \rightarrow 0}\left\{\limsup _{k \rightarrow \infty}\left[p_{i}^{\alpha}\left(m_{k}+\delta e_{i}^{\alpha}\right)-p_{i}^{\alpha}\left(m_{k}\right)\right] / \delta\right\}=\infty
$$

(3) If $C V$ is satisfied then for all $m \in \mathcal{M}^{c}:=R_{+}^{\mathcal{I} \times \mathcal{A}} \backslash \mathcal{M}, \forall i$ such that $\bar{m}_{i}=0, \forall \alpha \in \mathcal{A}_{i}$,

$$
\lim _{\epsilon \rightarrow 0}\left[p_{i}^{\alpha}\left(m+\epsilon e_{i}^{\alpha}\right)-p_{i}^{\alpha}(m)\right] / \epsilon=\infty
$$

Proof of Corollary 3: Recall that $p(m)$ was defined as the unique fixed point of the contraction map $F(m, \cdot)$. The iterates of this map are precisely the maps $G_{n}$ defined in Lemma 1; and, as well known, for every initial $p_{0}$ and for all $m, G_{n}\left(m, p_{0}\right)$ converges to $p(m)$. This convergence is even uniform in $m$, as all these contraction maps share the same contracting factor $K$ (see (3) of CIII ). Since $G_{n}$ is continuous in $m \in \mathcal{M}$, the continuity of $p(m)$ is immediate.

Since $G_{n}\left(m^{-\alpha}, m^{\alpha}, p\right)$ is $\alpha$-nondecreasing and $\alpha$-concave in $\left(m^{\alpha}, p\right)$, we get immediately that $G_{n, i}^{\alpha}\left(m^{-\alpha}, m^{\alpha}, p_{0}\right)$ is nondecreasing and concave in $m^{\alpha}$, for any fixed $m^{-\alpha}$ and $p_{0}$. Since non-decreasingness and concavity are preserved under point-wise convergence, (1) of the Corollary follows at once from Lemma 1.

We next prove (2). For all $i \in \mathcal{I}, \forall \alpha \in \mathcal{A}_{i}$, for all sequences $\left\{m_{k}\right\}_{k \in N} \subset \mathcal{M}$ such that $m_{k, i}^{\alpha} \leq \bar{m}_{k, i} / 2$ for all $k$ and $\bar{m}_{k, i} \rightarrow 0$ as $k \rightarrow \infty$, we infer from (P3) with $\hat{m}:=m_{k}+\delta e_{i}^{\alpha}$ and $m:=m_{k}$, that

$$
\left[G_{n, i}^{\alpha}\left(m_{k}+\delta e_{i}^{\alpha}, p_{0}^{\alpha}\right)-G_{n, i}^{\alpha}\left(m_{k}, p_{0}^{\alpha}\right)\right] / \delta \geq \inf _{q}\left[F_{i}^{\alpha}\left(m_{k}+\delta e_{i}^{\alpha}, q\right)-F_{i}^{\alpha}\left(m_{k}, q\right)\right] / \delta
$$

As $n \rightarrow \infty$, this yields:

$$
\left[p^{\alpha}\left(m_{k}+\delta e_{i}^{\alpha}\right)-p^{\alpha}\left(m_{k}\right)\right] / \delta \geq \inf _{q}\left[F_{i}^{\alpha}\left(m_{k}+\delta e_{i}^{\alpha}, q\right)-F_{i}^{\alpha}\left(m_{k}, q\right)\right] / \delta
$$

Taking the $\lim \sup _{k \rightarrow \infty}$ and then the $\lim _{\delta \rightarrow 0}$ preserves the above inequality, but by CIV the RHS goes to $\infty$. This proves (2).

Finally, let $m$ belong to $\mathcal{M}^{c}$, with $\bar{m}_{i}=0$. If $\alpha \in \mathcal{A}_{i}$, then taking $\hat{m}=m+\epsilon e_{i}^{\alpha}$ in (P3) for $G_{n}$ yields

$$
\left[G_{n, i}^{\alpha}\left(m+\epsilon e_{i}^{\alpha}, p_{0}^{\alpha}\right)-G_{n, i}^{\alpha}\left(m, p_{0}^{\alpha}\right)\right] / \epsilon \geq \inf _{q}\left[F_{i}^{\alpha}\left(m+\epsilon e_{i}^{\alpha}, q\right)-F_{i}^{\alpha}(m, q)\right] / \epsilon
$$


Letting $n$ go to $\infty$, we obtain:

$$
\left[p_{i}^{\alpha}\left(m+\epsilon e_{i}^{\alpha}\right)-p_{i}^{\alpha}(m)\right] / \epsilon \geq \inf _{q}\left[F_{i}^{\alpha}\left(m+\epsilon e_{i}^{\alpha}, q\right)-F_{i}^{\alpha}(m, q)\right] / \epsilon
$$

By CV , the RHS goes to $\infty$ as $\epsilon$ goes to 0 , proving (3).

Step 2: For $\epsilon>0$ and $b \geq \epsilon$, define the game $\Gamma^{\epsilon, b}$ by truncating the strategy sets of firms $\alpha$ to $[\epsilon, b]^{\mathcal{I}}$. Then $\Gamma^{\epsilon, b}$ has an NE.

Proof of Step 2: By Corollary 3, $p_{i}^{\alpha}\left(m^{\alpha}, m^{-\alpha}\right)$ is continuous in $m:=\left(m^{\alpha}, m^{-\alpha}\right) \in \mathcal{M}$ and concave in $m^{\alpha}$. Moreover $U^{\alpha}$ is continuous, concave and nondecreasing in all its variables by assumption CII and $C^{\alpha}$ is convex by CI . It follows that $\Pi^{\alpha}(m)=U^{\alpha}\left(p^{\alpha}\left(m^{\alpha}, m^{-\alpha}\right)\right)-C^{\alpha}\left(m^{\alpha}\right)$ is continuous in $m$ and concave in $m^{\alpha}$. The existence of NE now follows from the standard Nash argument [11].

Step 3: There exists $b^{*}>\epsilon^{*}>0$ such that NE of $\Gamma^{\epsilon, b^{*}}$ coincide with NE of $\Gamma^{\epsilon, \infty}$, for all $\epsilon<\epsilon^{*}$.

Proof of Step 3: By CI , $C^{\alpha}(x) \rightarrow \infty$ as $\|x\| \rightarrow \infty$ and $C^{\alpha}(\epsilon, \ldots, \epsilon) \rightarrow 0$ as $\epsilon \rightarrow 0$. Thus there exist scalars $0<\epsilon^{*}<b^{*}$ such that $-C^{\alpha}(\epsilon, \ldots, \epsilon)>U^{\alpha}(1,1, \ldots, 1)-U^{\alpha}(0,0, \ldots, 0)-C^{\alpha}\left(m^{\alpha}\right)$ whenever $\left\|m^{\alpha}\right\| \geq b$ and $\epsilon<\epsilon^{*}$. Any $\alpha$ with $\left\|m^{\alpha}\right\|>b^{*}$ would then benefit by unilaterally deviating from $m^{\alpha}$ to $(\epsilon, \ldots, \epsilon)$.

Step 4: Let $m(\epsilon)$ be an NE of $\Gamma^{\epsilon, b^{*}}$ and select a sequence $\epsilon_{n} \rightarrow 0$ so that $m\left(\epsilon_{n}\right) \rightarrow m$ as $n \rightarrow \infty$. Then $m$ belongs to $\mathcal{M}$ and is an NE of $\Gamma$.

Proof of Step 4: Suppose to the contrary that $\bar{m}_{i}=0$. Select a subsequence such that for some $\alpha \in \mathcal{A}_{i}$ $m_{i}^{\alpha}\left(\epsilon_{n}\right) \leq \bar{m}_{i}\left(\epsilon_{n}\right) / 2$ for all $n$. (Such an $\alpha$ exists since $\mathcal{A}_{i}$ has at least 2 elements.) Let $\zeta$ denote

$$
\zeta:=\frac{\zeta_{1}}{\zeta_{2}}
$$

where we have chosen $\zeta_{1}, \zeta_{2}>0$ with

$$
\zeta_{1}>\sup \left\{\frac{\partial_{+}}{\partial m_{i}^{\alpha}} C^{\alpha}\left(m^{\alpha}\right): m^{\alpha} \in\left[0,2 b^{*}\right]^{\mathcal{I}}\right\} \text { and } \zeta_{2}<\inf \left\{\frac{\partial_{+}}{\partial p_{i}^{\alpha}} U^{\alpha}\left(p^{\alpha}\right): p^{\alpha} \in[0,1]^{\mathcal{I}}, p_{i}^{\alpha}<3 / 4\right\}
$$

with $\partial_{+}$denoting the right hand derivative. (Clearly $\zeta$ is finite since $U^{\alpha}$ is concave and strictly increasing in $p_{i}^{\alpha}$ for $\alpha \in \mathcal{A}_{i}$ and since the convex function $C^{\alpha}$ is finite on $R_{+}^{\mathcal{I}}$.)

By (2) of Corollary 3 , there exists $\delta>0$ such that

$$
\limsup _{n \rightarrow \infty}\left[p_{i}^{\alpha}\left(m\left(\epsilon_{n}\right)+\delta e_{i}^{\alpha}\right)-p_{i}^{\alpha}\left(m\left(\epsilon_{n}\right)\right)\right] / \delta>\zeta
$$

By extracting a subsequence, we may suppose that $\forall n$

$$
\left[p_{i}^{\alpha}\left(m\left(\epsilon_{n}\right)+\delta e_{i}^{\alpha}\right)-p_{i}^{\alpha}\left(m\left(\epsilon_{n}\right)\right)\right] / \delta>\zeta
$$

Since $p_{j}^{\alpha}\left(m^{\alpha}, m^{-\alpha}\right)$ is nondecreasing in $m^{\alpha}$, we get $p^{\alpha}\left(m\left(\epsilon_{n}\right)+\delta e_{i}^{\alpha}\right) \geq p^{\alpha}\left(m\left(\epsilon_{n}\right)\right)+\zeta \delta e_{i}$ where $e_{i}$ denotes the vector in $R^{\mathcal{I}}$ whose $i$-th component is 1 and all others are 0 . Since $U^{\alpha}$ is nondecreasing, we get $U^{\alpha}\left(p^{\alpha}\left(m\left(\epsilon_{n}\right)+\delta e_{i}^{\alpha}\right)\right)-U^{\alpha}\left(p^{\alpha}\left(m\left(\epsilon_{n}\right)\right)\right) \geq U^{\alpha}\left(p^{\alpha}\left(m\left(\epsilon_{n}\right)\right)+\zeta \delta e_{i}\right)-U^{\alpha}\left(p^{\alpha}\left(m\left(\epsilon_{n}\right)\right)\right)>\zeta_{2} \zeta \delta=\zeta_{1} \delta$. Therefore the increase in utility via the unilateral deviation from $m\left(\epsilon_{n}\right)$ by firm $\alpha$ (when he increases his bid on customer $i$ in the amount $\delta$ ) is strictly more than $\zeta_{1} \delta$. But the incremental cost of this deviation is at most $\zeta_{1} \delta$. Thus the unilateral deviation is profitable, contradicting that $m\left(\epsilon_{n}\right)$ is an NE. 
Step 5: No $m \in \mathcal{M}^{c}$ can be an NE of $\Gamma$.

Proof of Step 5: Let $m$ be in $\mathcal{M}^{c}$, with $\bar{m}_{i}=0$. Let $\alpha$ be in $\mathcal{A}_{i}$ and consider the unilateral deviation from $m$ by $\alpha$ wherein he bids $\epsilon$ more on $i$. Since $p^{\alpha}$ is nondecreasing by claim 1 of Corollary 3 , we have that $p_{j}^{\alpha}\left(m+\epsilon e_{i}^{\alpha}\right) \geq p_{j}^{\alpha}(m)$, for all $j$, and thus $p^{\alpha}\left(m+\epsilon e_{i}^{\alpha}\right) \geq p^{\alpha}(m)+g(\epsilon) e_{i}$, where $g(\epsilon):=p_{i}^{\alpha}\left(m+\epsilon e_{i}^{\alpha}\right)-p_{i}^{\alpha}(m)$. Since $U^{\alpha}$ is nondecreasing and concave, we have, with $\zeta_{i}$ as in step 4:

$$
U^{\alpha}\left(p\left(m+\epsilon e_{i}^{\alpha}\right)\right) \geq U^{\alpha}\left(p(m)+g(\epsilon) e_{i}\right) \geq U^{\alpha}(p(m))+\zeta_{2} g(\epsilon)
$$

i.e., the gain in utility from the deviation is at least $\zeta_{2} g(\epsilon)$.

On the other hand, the cost incurred by the deviation is $C^{\alpha}\left(m^{\alpha}+\epsilon e_{i}\right)-C^{\alpha}\left(m^{\alpha}\right)$ which is at most $\zeta_{1} \epsilon$. Since $g(\epsilon) / \epsilon$ is greater that $\zeta$ for small enough $\epsilon$ (see (3) of Corollary 3), the deviation is profitable, and $m$ can thus not be an NE. This concludes the proof of Theorem 10 in the case when $Z^{\alpha}=R_{+}^{\mathcal{I}}$ for all $\alpha$.

Step 6: We next sketch the argument in the case where some $Z^{\beta}$ may be compact. For such $\beta$ 's, the expenditures $m^{\beta}$ are automatically bounded. (The condition " $C^{\beta}\left(m^{\beta}\right) \rightarrow \infty$ as $\left\|m^{\beta}\right\| \rightarrow \infty$ " was only invoked to bound $m^{\beta}$, and is now irrelevant). We shall need some obvious amendments in the argument of step 4 and step 5 .

First note that, by CII , there exists $\Delta>0$ such that $[0, \Delta]^{\mathcal{I}} \subset Z^{\alpha}$.

Consider as before a sequence $\left\{m\left(\epsilon_{n}\right)\right\}_{n \in N}$ of NE converging to $m$, with $i \in \mathcal{I}$ and $\alpha \in \mathcal{A}_{i}$ such that $m_{i}^{\alpha}\left(\epsilon_{n}\right) \leq \bar{m}_{i}\left(\epsilon_{n}\right) / 2 \rightarrow 0$ as $n \rightarrow \infty$ (and, $\epsilon_{n} \rightarrow 0$ ). Then either $m^{\alpha}\left(\epsilon_{n}\right)$ is in the interior of $Z^{\alpha}$ for infinitely many $n$, in which case the earlier argument holds word for word. We thus just have to consider the case where $m^{\alpha}\left(\epsilon_{n}\right)$ is on the boundary of $Z^{\alpha}$ for all $n$. In that case increasing the bid of firm $\alpha$ on $i$ may not be feasible, and we have to find other profitable unilateral deviations. To do so, let $\delta$ be as in step 5 ; and, since $\delta$ can be chosen arbitrarily small, let us assume that $0<\delta<\Delta$. Consider the sequence

$$
\tilde{m}_{n}^{\alpha}:=\left(1-t_{n}\right) m^{\alpha}\left(\epsilon_{n}\right)+t_{n}(\Delta, \cdots, \Delta)
$$

for $t_{n}=\delta /\left(\Delta-m_{i}^{\alpha}\left(\epsilon_{n}\right)\right)$. As $\left.m_{i}^{\alpha}\left(\epsilon_{n}\right)\right) \rightarrow 0, t_{n}$ belongs to $[0,1]$ for large enough $n$ and so $\tilde{m}_{n}^{\alpha}$ belongs to $Z^{\alpha}$ since $Z^{\alpha}$ is convex.

Now observe that for all $j$ where $m_{j}^{\alpha}<\Delta$, we have $\tilde{m}_{n, j}^{\alpha}>m_{j}^{\alpha}\left(\epsilon_{n}\right)$ for large $n$. Therefore, $p_{j}^{\alpha}$ is reduced after the unilateral deviation $\tilde{m}_{n}^{\alpha}$ only for those $j$ where $m_{j}^{\alpha} \geq \Delta$. The loss in utility caused by these reductions is bounded above by $D \delta$ for some constant $D$. But, as already argued in step 4, the gain in utility purely on account of the increase of $p_{i}^{\alpha}$ is an arbitrarily high multiple of $\delta$ as $n$ goes to $\infty$. This proves that the deviation is profitable for large enough $n$, contradicting that $m\left(\epsilon_{n}\right)$ is an NE for every $n$.

Step 5 can be argued in a similar fashion.

\subsection{Proof of Theorem 1}

First observe that on account on AI and AII, the quasi-linear model is a special case of our general model. So by Theorem 10, there exists an NE; and moreover if $m$ is an NE then ${ }^{19} \bar{m}_{i}>0$ for all $i \in \mathcal{I}$. It remains to show uniqueness of NE under assumption AIII.

\footnotetext{
${ }^{19}$ For better perspective, here is an alternative proof, valid only in the quasi-linear model, that $\bar{m}_{i}>0$. Suppose, to the contrary, that $\bar{m}_{i}=0$ for some $i$. By assumption, there exists $\alpha$ such that $u_{i}^{\alpha}>0$. By (6),

$$
v^{\alpha}=\left[u^{\alpha}\right]^{\top}\left(I-\Theta^{\alpha} W^{\alpha}\right)^{-1}\left(I-\Theta^{\alpha}\right)
$$
}


Suppose $m:=\left(m^{\alpha}\right)_{\alpha \in \mathcal{I}}$ and $\eta:=\left(\eta^{\alpha}\right)_{\alpha \in \mathcal{I}}$ are two NE's. Denote $r_{i}^{\alpha}:=m_{i}^{\alpha} / \bar{m}_{i}$ and $s_{i}^{\alpha}:=\eta_{i}^{\alpha} / \bar{\eta}_{i}$ (where, recall, $\bar{m}_{i}:=\sum_{\alpha \in \mathcal{A}} m_{i}^{\alpha}$ etc.). It suffices to show that $\bar{m}_{i}=\bar{\eta}_{i}$ and $r_{i}^{\alpha}=s_{i}^{\alpha}$ for all $\alpha \in \mathcal{A}$ and all $i \in \mathcal{I}$.

The first-order conditions ${ }^{20}$ for maximizing payoffs imply

$$
\begin{array}{r}
v_{i}^{\alpha} \lambda_{i}^{\alpha}\left(r_{i}^{\alpha}, \bar{m}_{i}\right)=c_{i}^{\alpha} \text { if } m_{i}^{\alpha}>0 \\
v_{i}^{\alpha} \lambda_{i}^{\alpha}\left(r_{i}^{\alpha}, \bar{m}_{i}\right) \leq c_{i}^{\alpha} \text { if } m_{i}^{\alpha}=0 \\
v_{i}^{\alpha} \lambda_{i}^{\alpha}\left(s_{i}^{\alpha}, \bar{\eta}_{i}\right)=c_{i}^{\alpha} \text { if } \eta_{i}^{\alpha}>0 \\
v_{i}^{\alpha} \lambda_{i}^{\alpha}\left(s_{i}^{\alpha}, \bar{\eta}_{i}\right) \leq c_{i}^{\alpha} \text { if } \eta_{i}^{\alpha}=0
\end{array}
$$

Fix $i \in \mathcal{I}$ and suppose w.l.o.g. that $\bar{m}_{i} \leq \bar{\eta}_{i}$.

Step 1: $s_{i}^{\alpha} \leq r_{i}^{\alpha}$ for all $\alpha \in \mathcal{A}$.

Proof: First note that, by (7), $v_{i}^{\alpha}=0$ implies $m_{i}^{\alpha}=0$ in any NE. Let $s_{i}^{\alpha}>0$ (otherwise the claim is vacuously true) and so we must have $v_{i}^{\alpha}>0$. Suppose, to the contrary, that $s_{i}^{\alpha}>r_{i}^{\alpha}$. Since $\bar{\eta}_{i} \geq \bar{m}_{i}$, assumption (2.1) on $\lambda_{i}^{\alpha}$ imply $\lambda_{i}^{\alpha}\left(s_{i}^{\alpha}, \bar{\eta}_{i}\right)<\lambda_{i}^{\alpha}\left(r_{i}^{\alpha}, \bar{m}_{i}\right)$. But (26), (27) and (28) and the fact that $v_{i}^{\alpha}>0$ together yield $\lambda_{i}^{\alpha}\left(s_{i}^{\alpha}, \bar{\eta}_{i}\right) \geq \lambda_{i}^{\alpha}\left(r_{i}^{\alpha}, \bar{m}_{i}\right)$, a contradiction.

Step 2: $s_{i}^{\alpha}=r_{i}^{\alpha}$ for all $\alpha \in \mathcal{A}$.

Proof: Immediate from step 1 , since $\sum_{\alpha \in \mathcal{A}} s_{i}^{\alpha}=1=\sum_{\alpha \in \mathcal{A}} r_{i}^{\alpha}$.

Step 3: $\bar{m}_{i}=\bar{\eta}_{i}$

Proof: Suppose $\bar{\eta}_{i}>\bar{m}_{i}$ (by assumption we already have $\geq$ ). By step 2, and assumption AIII, we have LHS of $(28)<$ LHS of (26). Since $\sum_{\beta \in \tau} r_{i}^{\beta}=1$ there exists $\beta^{\prime}$ such that $r_{i}^{\beta^{\prime}}>0$. By step $2, s_{i}^{\beta^{\prime}}=r_{i}^{\beta^{\prime}}$, so both (28) and (26) hold, hence LHS of (28) = LHS of (26), a contradiction. This proves step 3.

Since the choice of $i$ was arbitrary, we have shown that $\bar{\eta}_{i}=\bar{m}_{i}$ and $r_{i}^{\alpha}=s_{i}^{\alpha}$ for all $\alpha \in \mathcal{A}$ and all $i \in \mathcal{I}$. Thus $m=\eta$, establishing the uniqueness of NE.

\subsection{Proof of Theorem 2}

Note that $\lambda_{i}^{\alpha}\left(r_{i}^{\alpha}, \bar{m}_{i}\right)=\left(1-r_{i}^{\alpha}\right) / \bar{m}_{i}$ in our canonical case. Thus the first-order conditions (26) and (27) become

$$
\begin{aligned}
\frac{1-r_{i}^{\alpha}}{\bar{m}_{i}} & =\kappa_{i}^{\alpha} \text { if } r_{i}^{\alpha}>0 \\
\frac{1}{\bar{m}_{i}} & \leq \kappa_{i}^{\alpha} \text { if } r_{i}^{\alpha}=0
\end{aligned}
$$

Since $\bar{m}_{i}>0$ at an NE, at least one firm spends positive money on $i$. Let $l_{i}$ denote the maximal $\alpha$ such that $r_{i}^{\alpha}>0$. Then observe that, if $r_{i}^{\alpha}>0$, then $\kappa_{i}^{\beta} \leq \kappa_{i}^{\alpha}$ implies $r_{i}^{\beta}>0$. Therefore $r_{i}^{\alpha}>0$ for $\alpha \leq l_{i}$ and $r_{\alpha}=0$

$$
=\left[u^{\alpha}\right]^{\top} \sum_{n=0}^{\infty}\left(\Theta^{\alpha} W^{\alpha}\right)^{n}\left(I-\Theta^{\alpha}\right)
$$

from which it follows that $[u]^{\top}$ is being multiplied by a matrix with non negative entries and strictly positive diagonal entries. Hence $v_{i}^{\alpha}>0$.

Let firm $\alpha$ unilaterally deviate from $m$ by spending a small $\delta$ on customer $i$. By (7), his change in payoff is

$$
v_{i}^{\alpha} \gamma_{i}^{\alpha}(\delta, 0)-c_{i}^{\alpha} \delta
$$

which, using (3) of AII, becomes positive for small enough $\delta$, contradicting that $m$ is an NE. We conclude that $\bar{m}_{i}>0$ for all $i \in \mathcal{I}$.

${ }^{20}$ Since $\bar{m}_{i}>0$ and $\bar{\eta}_{i}>0$, and the $\gamma_{i}^{\alpha}$ are differentiable away from zero, these conditions can be invoked. 
for $\alpha>l_{i}$. Therefore $\bar{m}_{i}=\sum_{\beta=1}^{l_{i}} m_{i}^{\beta}$, and $1=\sum_{\beta=1}^{l_{i}} r_{i}^{\beta}$. Thus summing (30) for $\alpha \leq l_{i}$ yields:

$$
\sum_{\alpha=1}^{l_{i}} \kappa_{i}^{\alpha}=\sum_{\alpha=1}^{l_{i}} \frac{1-r_{i}^{\alpha}}{\bar{m}_{i}}=\frac{l_{i}-\sum_{\alpha=1}^{l_{i}} r_{i}^{\alpha}}{\bar{m}_{i}}=\frac{l_{i}-1}{\bar{m}_{i}}
$$

Thus,

$$
\bar{m}_{i}=\frac{l_{i}-1}{\sum_{\beta=1}^{l_{i}} \kappa_{i}^{\beta}}
$$

and $1-\kappa_{i}^{l_{i}} \bar{m}_{i}=r_{i}^{l_{i}}>0$, which implies that $\sum_{\beta=1}^{l_{i}} \kappa_{i}^{\beta}>\kappa_{i}^{l_{i}}\left(l_{i}-1\right)$. On the other hand, if $l_{i}<|\mathcal{I}|$, then we must have $1 \leq \kappa_{i}^{l_{i}+1} \bar{m}_{i}$, and thus $\sum_{\beta=1}^{l_{i}} \kappa_{i}^{\beta} \leq \kappa_{i}^{l_{i}+1}\left(l_{i}-1\right)$. So $l_{i}$ must be the maximal $l$ such that $\sum_{\beta=1}^{l} \kappa_{i}^{\beta}>\kappa_{i}^{l}\left(l_{i}-1\right)$. Once $l_{i}$ is known, we get the NE actions as follows: For $\alpha \leq l_{i}$ :

$$
m_{i}^{\alpha}=r_{i}^{\alpha} \bar{m}_{i}=\left(1-\kappa_{i}^{\alpha} \bar{m}_{i}\right) \bar{m}_{i}=\left(1-\frac{\kappa_{i}^{\alpha}\left(l_{i}-1\right)}{\sum_{\beta=1}^{l_{i}} \kappa_{i}^{\beta}}\right)\left(\frac{l_{i}-1}{\sum_{\beta=1}^{l_{i}} \kappa_{i}^{\beta}}\right)
$$

and, for $\alpha>l_{i}$, we must have $m_{i}^{\alpha}=0$.

\subsection{Proof of Theorem 4}

It will be convenient to create a micro-model of how the decision $L_{t} \in\{$ Stop, Move $\}$ is taken in our Markov chain. Before starting the Markov chain, one can, for each state $i$, consider an infinite sequence of independent decisions $\left\{L_{k}^{i}\right\}_{k=0,1,2, \ldots}$, with $\operatorname{prob}\left(L_{k}^{i}=\right.$ Move $)=\theta_{i}^{\alpha}(\theta)$. Each time the process comes to state $i$ the decision to Stop or to Move is taken according to the first unused decision $L_{k}^{i}$. In other words, if $N_{t}^{i}$ denotes the number of visits of state $i$ up to time $t$, we get $L_{t}=L_{N_{t}^{i_{t}}}^{i_{t}}$.

Let then $K_{i}$ denote the smallest $k$ such that $L_{k}^{i}=S t o p$. Clearly $K_{i}$ is a geometric random variable with parameter $\theta_{i}^{\alpha}(\theta)$, so that, for an integer $k, \operatorname{prob}\left(K_{i}=k\right)=\left(1-\theta_{i}^{\alpha}\right)\left(\theta_{i}^{\alpha}\right)^{k}$ and $P\left(K_{i}>k\right)=\left(\theta_{i}^{\alpha}\right)^{k+1}$.

The event $\left\{i_{T}=i\right\}$ coincides then with $\left\{\exists t \mid N_{t}^{i} \geq K_{i} \& N_{t}^{j}<K_{j}, \forall j \neq i\right\}$.

The ergodic Theorem (See e.g. [4]) tells us that, as $t$ goes to $\infty, n_{t}^{i}:=N_{t}^{i} / t$ converges almost surely to the random variable $E_{\mu^{\alpha}}\left[\mathbb{1}_{i}\right]=\mu_{i}^{\alpha}$, where $\mu^{\alpha}$ is the unique invariant measure $\left(\mathcal{I}=I_{1}\right)$.

Therefore, for all $\epsilon>0$, there exists $N$ such that $\operatorname{prob}(A)>1-\epsilon$, where $A:=\left\{\forall t>N, \forall i \in \mathcal{I}:\left|\mu_{i}^{\alpha}-n_{t}^{i}\right|<\epsilon\right\}$. Define also $B:=\left\{K_{i}>N\right\}$. Then

$$
\begin{aligned}
\operatorname{prob}\left(i_{T}=i\right) & \geq \operatorname{prob}\left(\left\{i_{T}=i\right\} \cap A \cap B\right) \\
& =\operatorname{prob}\left(\left\{\exists t \mid t n_{t}^{i} \geq K_{i} \& t n_{t}^{j}<K_{j}, \forall j \neq i\right\} \cap A \cap B\right) \\
& \geq \operatorname{prob}\left(\left\{\exists t \mid t\left(\mu_{i}^{\alpha}-\epsilon\right) \geq K_{i} \& t\left(\mu_{j}^{\alpha}+\epsilon\right)<K_{j}, \forall j \neq i\right\} \cap A \cap B\right) \\
& \geq \operatorname{prob}\left(\left\{\frac{K_{i}}{\mu_{i}^{\alpha}-\epsilon}<\frac{K_{j}}{\mu_{j}^{\alpha}+\epsilon}, \forall j \neq i\right\}\right)-\operatorname{prob}\left(A^{c}\right)-\operatorname{prob}\left(B^{c}\right)
\end{aligned}
$$

Since $\frac{\mu_{i}^{\alpha}-\epsilon}{\mu_{j}^{\alpha}+\epsilon}$ is decreasing in $\epsilon$,

$$
\lim _{\epsilon \rightarrow 0} \operatorname{prob}\left(\left\{\frac{K_{i}}{\mu_{i}^{\alpha}-\epsilon}<\frac{K_{j}}{\mu_{j}^{\alpha}+\epsilon}, \forall j \neq i\right\}\right)=\operatorname{prob}\left(\left\{\frac{K_{i}}{\mu_{i}^{\alpha}}<\frac{K_{j}}{\mu_{j}^{\alpha}}, \forall j \neq i\right\}\right) .
$$

Therefore

$$
\operatorname{prob}\left(i_{T}=i\right) \geq \operatorname{prob}\left(\left\{\frac{K_{i}}{\mu_{i}^{\alpha}}<\frac{K_{j}}{\mu_{j}^{\alpha}}, \forall j \neq i\right\}\right)-\operatorname{prob}\left(B^{c}\right)
$$


Next, with $[x]$ being the integer part of the real number $x$, we get

$$
\begin{aligned}
\operatorname{prob}\left(\left\{\frac{K_{i}}{\mu_{i}^{\alpha}}<\frac{K_{j}}{\mu_{j}^{\alpha}}, \forall j \neq i\right\}\right) & =\sum_{k=0}^{\infty}\left(1-\theta_{i}^{\alpha}\right)\left(\theta_{i}^{\alpha}\right)^{k} \prod_{j \neq i}\left(\theta_{j}^{\alpha}\right)^{\left[\frac{\mu_{j}^{\alpha}}{\mu_{i}^{\alpha}} k\right]+1} \\
& \geq\left(\prod_{j \neq i} \theta_{j}^{\alpha}\right)\left(1-\theta_{i}^{\alpha}\right) \sum_{k=0}^{\infty}\left(\prod_{j \in \mathcal{I}}\left(\theta_{j}^{\alpha}\right)^{\frac{\mu_{j}^{\alpha}}{\mu_{i}^{\alpha}}}\right)^{k} \\
& =\left(\prod_{j \neq i} \theta_{j}^{\alpha}\right) \frac{1-\theta_{i}^{\alpha}}{1-\prod_{j \in \mathcal{I}}\left(\theta_{j}^{\alpha}\right)^{\frac{\mu_{j}^{\alpha}}{\mu_{i}^{\alpha}}}}
\end{aligned}
$$

Using (12) and (14), the limit of this RHS as $\theta \rightarrow 1$ is

$$
\frac{\delta_{i}^{\alpha} \mu_{i}^{\alpha}}{\sum_{j \in \mathcal{I}} \delta_{j}^{\alpha} \mu_{j}^{\alpha}}
$$

Similarly,

$$
\begin{aligned}
\operatorname{prob}\left(i_{T}=i\right) & \leq \operatorname{prob}\left(\left\{i_{T}=i\right\} \cap A \cap B\right)+\operatorname{prob}\left(A^{c}\right)+\operatorname{prob}\left(B^{c}\right) \\
& =\operatorname{prob}\left(\left\{\exists t \mid t n_{t}^{i} \geq K_{i} \& t n_{t}^{j}<K_{j}, \forall j \neq i\right\} \cap A \cap B\right)+\operatorname{prob}\left(A^{c}\right)+\operatorname{prob}\left(B^{c}\right) \\
& \leq \operatorname{prob}\left(\left\{\exists t \mid t\left(\mu_{i}^{\alpha}+\epsilon\right) \geq K_{i} \& t\left(\mu_{j}^{\alpha}-\epsilon\right)<K_{j}, \forall j \neq i\right\}\right)+\operatorname{prob}\left(A^{c}\right)+\operatorname{prob}\left(B^{c}\right) \\
& \leq \operatorname{prob}\left(\left\{\frac{K_{i}}{\mu_{i}^{\alpha}+\epsilon} \leq \frac{K_{j}}{\mu_{j}^{\alpha}-\epsilon}, \forall j \neq i\right\}\right)+\operatorname{prob}\left(A^{c}\right)+\operatorname{prob}\left(B^{c}\right)
\end{aligned}
$$

Letting $\epsilon$ go to 0 yields

$$
\operatorname{prob}\left(i_{T}=i\right) \leq \operatorname{prob}\left(\left\{\frac{K_{i}}{\mu_{i}^{\alpha}} \leq \frac{K_{j}}{\mu_{j}^{\alpha}}, \forall j \neq i\right\}\right)+\operatorname{prob}\left(B^{c}\right)
$$

But

$$
\begin{aligned}
\operatorname{prob}\left(\left\{\frac{K_{i}}{\mu_{i}^{\alpha}} \leq \frac{K_{j}}{\mu_{j}^{\alpha}}, \forall j \neq i\right\}\right) & =\sum_{k=0}^{\infty}\left(1-\theta_{i}^{\alpha}\right)\left(\theta_{i}^{\alpha}\right)^{k} \prod_{j \neq i}\left(\theta_{j}^{\alpha}\right)^{\left[\frac{\mu_{j}^{\alpha}}{\mu_{i}^{\alpha}} k\right]} \\
& \leq\left(\prod_{j \neq i} \theta_{j}^{\alpha}\right)^{-1}\left(1-\theta_{i}^{\alpha}\right) \sum_{k=0}^{\infty}\left(\prod_{j \in \mathcal{I}}\left(\theta_{j}^{\alpha}\right)^{\frac{\mu_{j}^{\alpha}}{\mu_{i}^{\alpha}}}\right)^{k} \\
& =\left(\prod_{j \neq i} \theta_{j}^{\alpha}\right)^{-1} \frac{1-\theta_{i}^{\alpha}}{1-\prod_{j \in \mathcal{I}}\left(\theta_{j}^{\alpha}\right)^{\frac{\mu_{j}^{\alpha}}{\mu_{i}^{\alpha}}}}
\end{aligned}
$$

As $\theta$ goes to 1 , this also converges to (33), which therefore is also the limit of $\operatorname{prob}\left(i_{T}=i\right)$.

\subsection{Proof of Theorem 5}

Eq. (6), and the fact that $W$ is sub-stochastic and the entries in $\Theta$ are between 0 and 1, imply

$$
v:=[u]^{\top}(I-\Theta W)^{-1}(I-\Theta)=[u]^{\top}\left(I+\sum_{k=1}^{\infty}(\Theta W)^{k}\right)(I-\Theta)
$$

Let $\tilde{v}$ be defined in an analogous manner, using $\tilde{W}$ in place of $W$. Using the fact that (i) $u \gg 0$, (ii) $(I-\Theta)$ is a non-negative diagonal matrix and (iii) $\tilde{W}>W$, it follows that $\tilde{v} \geq v$ and $\tilde{v}_{j}>v_{j}$. Eq. (11) now implies that in the new unique NE, every active firm will spend more money on customer $j$ and no less on the other customers. (Note that the set of active and blockaded firms remain unchanged we replace $W$ by $\tilde{W}$, since these sets depend only on the $\operatorname{costs} c^{\alpha}$ of firms $\alpha$, as shown in Section 3.3.)

We next claim that

$$
\frac{\tilde{v}_{j}}{\tilde{v}_{k}} \geq \frac{v_{j}}{v_{k}}
$$


for all $k \in \mathcal{I}$. Indeed, let $\bar{u}$ denote $\bar{u}:=\sum_{i} u_{i}$ and let $\mu$ be defined as $\mu:=u / \bar{u}$. Let $X$ and $\tilde{X}$ denote the sub-Markovian matrices in $R^{\mathcal{I}^{\prime} \times \mathcal{I}^{\prime}}$, with $\mathcal{I}^{\prime}=\mathcal{I} \cup\{w\}$ :

$$
X:=\left(\begin{array}{cc}
\Theta W & 0 \\
{[\mu]^{\top}} & 0
\end{array}\right) \text { and } \tilde{X}:=\left(\begin{array}{cc}
\Theta \tilde{W} & 0 \\
{[\mu]^{\top}} & 0
\end{array}\right) .
$$

It is then easily checked that

$$
X^{n}=\left(\begin{array}{cc}
(\Theta W)^{n} & 0 \\
{[\mu]^{\top}(\Theta W)^{n-1}} & 0
\end{array}\right) \text { and } \tilde{X}^{n}=\left(\begin{array}{cc}
(\Theta \tilde{W})^{n} & 0 \\
{[\mu]^{\top}(\Theta \tilde{W})^{n-1}} & 0
\end{array}\right) .
$$

It results from the definitions of $v, \tilde{v}$ and $\mu$ that $a:=v(I-\Theta)^{-1} / \bar{u}=[\mu]^{\top} \sum_{k=0}^{\infty}(\Theta W)^{k}$ and $\tilde{a}:=\tilde{v}(I-\Theta)^{-1} / \bar{u}=$ $[\mu]^{\top} \sum_{k=0}^{\infty}(\Theta \tilde{W})^{k}$. Therefore, setting, $Y:=\sum_{n=0}^{\infty} X^{n}$ and $\tilde{Y}=\sum_{n=0}^{\infty} \tilde{X}^{n}$, we get

$$
Y=\left(\begin{array}{cc}
\sum_{n=0}^{\infty}(\Theta W)^{n} & 0 \\
a & 0
\end{array}\right) \text { and } \tilde{Y}=\left(\begin{array}{cc}
\sum_{n=0}^{\infty}(\Theta \tilde{W})^{n} & 0 \\
\tilde{a} & 0
\end{array}\right) \text {. }
$$

Theorem 1 in [14], on resolvent nonnegative matrices, states that, $\forall k \in \mathcal{I}, \frac{\tilde{a}_{j}}{\tilde{a}_{k}}=\frac{\tilde{Y}_{w, j}}{\tilde{Y} w, k} \geq \frac{Y_{w, j}}{Y w, k}=\frac{a_{j}}{a_{k}}$. Therefore

$$
\frac{\tilde{v}_{j}}{\tilde{v}_{k}} \frac{\left(1-\theta_{k}\right)}{\left(1-\theta_{j}\right)}=\frac{\tilde{a}_{j}}{\tilde{a}_{k}} \geq \frac{a_{j}}{a_{k}}=\frac{v_{j}}{v_{k}} \frac{\left(1-\theta_{k}\right)}{\left(1-\theta_{j}\right)},
$$

and (34) follows.

Using Eq. (11) and (34) we get

$$
\frac{\tilde{m}_{j}^{\alpha}}{\tilde{m}_{k}^{\alpha}} \geq \frac{m_{j}^{\alpha}}{m_{k}^{\alpha}}
$$

for all active firms $\alpha$ and $k \in \mathcal{I}$. In other words, as the influence of customer $j$ is enhanced in the social network, the money spent on him by active firms will go up not just in absolute, but also relative, terms.

\subsection{Proof of Theorem 6}

The proof is based on the following Lemma which may be of independent interest. Let $B$ be an $\mathcal{I} \times \mathcal{A}$ matrix with entries $B_{i}^{\alpha}$ for $i \in \mathcal{I}$ and $\alpha \in \mathcal{A}$. Define $\bar{B}^{\alpha}:=\sum_{i} B_{i}^{\alpha}$ and $\bar{B}_{i}:=\sum_{\alpha} B_{i}^{\alpha}$. A cell $(i, \alpha)$ is said to be positive for $B$ if $\bar{B}_{i} \geq 0, \bar{B}^{\alpha} \geq 0$ and $B_{i}^{\alpha}>0$. It is said to be negative for $B$ if it is positive for $-B$. Finally it is said to be signed if it is either positive or negative for $B$.

Lemma 2 Any non zero matrix $B$ has a signed cell.

Proof of Lemma 2 Suppose $B$ has no signed cell. Let $\mathcal{A}^{+}:=\left\{\alpha \in \mathcal{A}: \bar{B}^{\alpha} \geq 0\right\}, \mathcal{A}^{-}:=\mathcal{A} \backslash \mathcal{A}^{+}$, and similarly $\mathcal{I}^{+}:=\left\{i \in \mathcal{I}: \bar{B}_{i} \geq 0\right\}, \mathcal{I}^{-}:=\mathcal{I} \backslash \mathcal{I}^{+}$.

Since $B$ has no positive cell,

$$
B_{i}^{\alpha} \leq 0 \text { for all } \alpha \in \mathcal{A}^{+} \text {and } i \in \mathcal{I}^{+}
$$

Similarly, since $B$ has no negative cell,

$$
B_{i}^{\alpha} \geq 0 \text { for all } \alpha \in \mathcal{A}^{-} \text {and } i \in \mathcal{I}^{-}
$$

Now, for all $\alpha \in \mathcal{A}^{-}, 0>\bar{B}^{\alpha}=\sum_{\mathcal{I}^{+}} B_{i}^{\alpha}+\sum_{\mathcal{I}^{-}} B_{i}^{\alpha}$. By (36) this is only possible if $\sum_{\mathcal{I}^{+}} B_{i}^{\alpha}<0$. 
We will prove that $\mathcal{A}^{-}=\emptyset$. Indeed, if $\mathcal{A}^{-} \neq \emptyset$, then

$$
\sum_{\mathcal{A}^{-}} \sum_{\mathcal{I}^{+}} B_{i}^{\alpha}<0
$$

On the other hand, for all $i \in \mathcal{I}^{+}$we have $0 \leq \bar{B}_{i}=\sum_{\mathcal{A}^{+}} B_{i}^{\alpha}+\sum_{\mathcal{A}^{-}} B_{i}^{\alpha}$. By (35) this is only possible if $\sum_{\mathcal{A}^{-}} B_{i}^{\alpha} \geq 0$ and therefore $\sum_{\mathcal{A}^{-}} \sum_{\mathcal{I}^{+}} B_{i}^{\alpha} \geq 0$, a contradiction.

A symmetric argument shows that $\mathcal{I}^{-}=\emptyset$. So, by (35), all elements $B_{i}^{\alpha}$ are non-positive and their row sums $\bar{B}_{i}$ are non-negative. This is only possible if $B=0$.

Now we complete the proof of Theorem 6. Let $m \in R^{\mathcal{I} \times \mathcal{A}}$ and $\eta \in R^{\mathcal{I} \times \mathcal{A}}$ be two NE of the game. Define the matrix $B=\eta-m$. We shall show that it has no signed cell, so that by the Lemma, $B=0$, proving $m=\eta$.

Suppose $(i, \alpha)$ is a positive cell for $B$, then we have $m_{i}^{\alpha}<\eta_{i}^{\alpha}, \bar{m}_{i} \leq \bar{\eta}_{i}, \bar{m}^{\alpha} \leq \bar{\eta}^{\alpha}$. By the strictly decreasing property of $h_{i}^{\alpha}$ in its first variable, if $x \in h_{i}^{\alpha}\left(m_{i}^{\alpha}, \bar{m}_{i}, \bar{m}^{\alpha}\right)$ and $y \in h_{i}^{\alpha}\left(\eta_{i}^{\alpha}, \bar{\eta}_{i}, \bar{\eta}^{\alpha}\right)$, we must have $x>y$. But this contradicts the first order conditions of NE, according to which $0 \in h_{i}^{\alpha}\left(m_{i}^{\alpha}, \bar{m}_{i}, \bar{m}^{\alpha}\right)$ and $0 \in h_{i}^{\alpha}\left(\eta_{i}^{\alpha}, \bar{\eta}_{i}, \bar{\eta}^{\alpha}\right)$, $\forall i, \alpha$.

By a symmetric argument, $B$ has no negative cell.

\subsection{Proof of Theorem 7}

By Corollary 2, we need only verify that, if $m$ is an NE, then $m \in \Omega$, i.e., $r_{i}^{\alpha} \leq 1 / 2$ for all $\alpha$ and $i$. Suppose some $r_{i}^{\alpha}>1 / 2$. Then, clearly, there exists $\beta \in \mathcal{A}^{\alpha}(n)$ such that $r_{i}^{\beta}<1 / 2 n$. We further have:

Claim 1 There exists $j \in \mathcal{I} \backslash\{i\}$ such that $r_{j}^{\beta}>r_{j}^{\alpha}$.

Proof: It suffices to show that $\beta$ has more money left to spend ${ }^{21}$ on $\mathcal{I} \backslash\{i\}$ than does $\alpha$, namely:

$$
M^{\alpha}-m_{i}^{\alpha}<M^{\beta}-m_{i}^{\beta}
$$

i.e.,

$$
m_{i}^{\alpha}-m_{i}^{\beta}>M^{\alpha}-M^{\beta}
$$

Now

$$
\begin{aligned}
m_{i}^{\alpha}-m_{i}^{\beta} & =\left(r_{i}^{\alpha}-r_{i}^{\beta}\right) \bar{m}_{i} \\
& >\left(\frac{1}{2}-\frac{1}{2 n}\right) \bar{m}_{i} \\
& =\left(\frac{n-1}{2 n}\right) \bar{m}_{i} \\
& \geq \frac{1}{4} \bar{m}_{i}
\end{aligned}
$$

Consider any $k \in \mathcal{I} \backslash\{i\}$ such that $r_{k}^{\beta}>0$ (we shall deal shortly with the case that no such $k$ exists). The first order conditions of NE imply

$$
v_{i}^{\beta}\left(\frac{1-r_{i}^{\beta}}{\bar{m}_{i}}\right) \leq v_{k}^{\beta}\left(\frac{1-r_{k}^{\beta}}{\bar{m}_{k}}\right)
$$

${ }^{21}$ Since, $v_{j}^{\beta}>0$ for some $j \in \mathcal{I}$, it follows that $\sum_{j \in \mathcal{I}} m_{j}^{\beta}=M^{\beta}$. 
Hence

$$
\begin{aligned}
\bar{m}_{i} & \geq \frac{v_{i}^{\beta}}{v_{k}^{\beta}}\left(\frac{1-r_{i}^{\beta}}{1-r_{k}^{\beta}}\right) \bar{m}_{k} \\
& \geq\left(v_{\min }^{\beta} / v_{\max }^{\beta}\right)\left(\frac{1-1 / 2 n}{1-0}\right) \bar{m}_{k} \\
& \geq\left(v_{\min }^{\beta} / v_{\max }^{\beta}\right) \frac{3}{4} \bar{m}_{k}
\end{aligned}
$$

Summing over all $k$ such that $r_{k}^{\beta}>0$, we get

$$
|\mathcal{I}| \bar{m}_{i} \geq \frac{3}{4}\left(v_{\min }^{\beta} / v_{\max }^{\beta}\right) M^{\beta}
$$

i.e.,

$$
\bar{m}_{i} \geq \frac{3}{4|\mathcal{I}|}\left(v_{\min }^{\beta} / v_{\max }^{\beta}\right) M^{\beta}
$$

(When $r_{k}^{\beta}=0$ for all $k \in \mathcal{I} \backslash\{i\}$, we must have $\bar{m}_{i} \geq M^{\beta}$ and therefore the above inequality still holds.)

This inequality along with (38), implies

$$
\begin{aligned}
m_{i}^{\alpha}-m_{i}^{\beta} & >\frac{1}{4} \frac{3}{4|\mathcal{I}|}\left(v_{\min }^{\beta} / v_{\max }^{\beta}\right) M^{\beta} \\
& =\frac{3}{16|\mathcal{I}|}\left(v_{\min }^{\beta} / v_{\max }^{\beta}\right) M^{\beta}
\end{aligned}
$$

On the other hand, condition (iii) of Theorem 7 says

$$
M^{\alpha}-M^{\beta} \leq \frac{3}{16|\mathcal{I}|}\left(v_{\min }^{\beta} / v_{\max }^{\beta}\right) M^{\beta}
$$

Therefore

$$
m_{i}^{\alpha}-m_{i}^{\beta}>M^{\alpha}-M^{\beta}
$$

establishing the claim.

The first-order conditions of NE imply that (21) and (22) hold, with $\beta$ substituted for $\tilde{\alpha}$. This yields ${ }^{22}$

$$
\frac{v_{i}^{\alpha} v_{j}^{\beta}}{v_{i}^{\beta} v_{j}^{\alpha}} \geq \frac{\left(1-r_{j}^{\alpha}\right)\left(1-r_{i}^{\beta}\right)}{\left(1-r_{j}^{\beta}\right)\left(1-r_{i}^{\alpha}\right)} \geq \frac{1-1 / 2 n}{1 / 2}=\frac{2 n-1}{n}
$$

Using condition (ii)(b) of Theorem 7, we get

$$
k_{1} k_{2} \geq \frac{2 n-1}{n}
$$

which contradicts condition (ii)(a). We conclude that $r_{i}^{\alpha} \leq 1 / 2$ for every $\alpha$ and $i$, proving the Theorem.

\footnotetext{
${ }^{22}$ Recalling that (a) $r_{j}^{\beta}>r_{j}^{\alpha}, r_{i}^{\alpha}>1 / 2, r_{i}^{\beta}<1 / 2 n$; (b) $r_{j}^{\beta}<1$ since at least two companies bid on any customer-node in an NE; (c) by (ii) (and the obvious fact that a company bids money on a node only if it places positive value on it) we have $v_{i}^{\alpha}>0, v_{i}^{\beta}>0, v_{j}^{\alpha}>0, v_{j}^{\beta}>0$; (d) both $\bar{m}_{i}$ and $\bar{m}_{j}$ are positive by Theorem 10 . These conditions together imply that the LHS and RHS of (21) and (22) are all positive.
} 


\subsection{Proof of Theorem 8}

Let $m$ be an NE. As argued in the proof of Theorem $3, \bar{m}_{i}>0$ for all $i \in \mathcal{I}$, so that the derivatives $\lambda_{i}^{\alpha}\left(r_{i}^{\alpha}, \bar{m}_{i}\right)$ are well defined.

Step 1: $r_{i}^{\alpha}=r_{j}^{\alpha}:=r^{\alpha}$ for all $i \in \mathcal{I}, j \in \mathcal{I}$ and $\alpha \in \mathcal{A}$.

Proof: Suppose $r_{i}^{\alpha}>r_{j}^{\alpha}$ for some $\alpha, i, j$. Since $\sum_{\beta \in \mathcal{A}} r_{i}^{\beta}=1=\sum_{\beta \in \mathcal{A}} r_{j}^{\beta}$, there exists $\beta$ such that $r_{i}^{\beta}<r_{j}^{\beta}$. Since $r_{i}^{\alpha}>0$, the first-order conditions for $\alpha$ at $i$ and $j$ are $\left(\right.$ where $\left.\xi^{\alpha}\left(\bar{m}^{\alpha}\right)=\left(d / d \bar{m}^{\alpha}\right) C^{\alpha}\left(\bar{m}^{\alpha}\right)\right)$

$$
v_{i} \frac{\lambda^{\alpha}\left(r_{i}^{\alpha}\right)}{f_{i}\left(\bar{m}_{i}\right)}=\xi^{\alpha}\left(\bar{m}^{\alpha}\right)
$$

and

which gives

$$
v_{j} \frac{\lambda^{\alpha}\left(r_{j}^{\alpha}\right)}{f_{j}\left(\bar{m}_{j}\right)} \leq \xi^{\alpha}\left(\bar{m}^{\alpha}\right)
$$

$$
\frac{v_{i} \lambda^{\alpha}\left(r_{i}^{\alpha}\right)}{f_{i}\left(\bar{m}_{i}\right)} \geq \frac{v_{j} \lambda^{\alpha}\left(r_{j}^{\alpha}\right)}{f_{j}\left(\bar{m}_{j}\right)}
$$

Similarly

$$
\frac{v_{i} \lambda^{\beta}\left(r_{i}^{\beta}\right)}{f_{i}\left(\bar{m}_{i}\right)} \leq \frac{v_{j} \lambda^{\beta}\left(r_{j}^{\beta}\right)}{f_{j}\left(\bar{m}_{j}\right)}
$$

From (39) and (40) we obtain

$$
\frac{\lambda^{\alpha}\left(r_{i}^{\alpha}\right)}{\lambda^{\alpha}\left(r_{j}^{\alpha}\right)} \geq \frac{\lambda^{\beta}\left(r_{i}^{\beta}\right)}{\lambda^{\beta}\left(r_{j}^{\beta}\right)}
$$

But, by (BI), LHS of $(41)<1$ and RHS of $(41)>1$, a contradiction.

Let $\eta$ be another NE and define $s_{i}^{\alpha}:=\eta_{i}^{\alpha} / \bar{\eta}_{i}$. As shown in Step $1, s_{i}^{\alpha}=s_{j}^{\alpha}:=s^{\alpha}$ for all $\alpha \in \mathcal{A}$ and $i, j \in \mathcal{I}$. Step 2: $r^{\alpha}=s^{\alpha}$ for all $\alpha \in \mathcal{A}$.

Proof: Suppose not. W.l.o.g. let $\bar{m} \geq \bar{\eta}$. Clearly there exists $i \in \mathcal{I}$ such that $\bar{m}_{i} \geq \bar{\eta}_{i}$ and (since $\sum_{\beta} \in \mathcal{A} r^{\beta}=$ $\left.1=\sum_{\beta \in \mathcal{A}} s^{\beta}\right)$ there exists $\alpha \in \mathcal{A}$ such that $r^{\alpha}>s^{\alpha} \geq 0$. The first order conditions give the following:

$$
\begin{aligned}
& \frac{v_{i} \lambda^{\alpha}\left(r^{\alpha}\right)}{f_{i}\left(\bar{m}_{i}\right)}=\xi^{\alpha}\left(r^{\alpha} \bar{m}\right) \\
& \frac{v_{i} \lambda^{\alpha}\left(s^{\alpha}\right)}{f_{i}\left(\bar{\eta}_{i}\right)} \leq \xi^{\alpha}\left(s^{\alpha} \bar{\eta}\right)
\end{aligned}
$$

By the convexity of the cost function and the fact that $r^{\alpha}>s^{\alpha}$ and $\bar{m} \geq \bar{\eta}$, RHS of (42) $\geq$ RHS of (43), so

$$
\frac{\lambda^{\alpha}\left(r^{\alpha}\right)}{f_{i}\left(\bar{m}_{i}\right)} \geq \frac{\lambda^{\alpha}\left(s^{\alpha}\right)}{f_{i}\left(\bar{\eta}_{i}\right)}
$$

But, since $r^{\alpha}>s^{\alpha}$ and $\bar{m}_{i} \geq \bar{\eta}_{i}$, (BI) implies $\lambda^{\alpha}\left(r^{\alpha}\right)<\lambda^{\alpha}\left(s^{\alpha}\right)$ and $f_{i}\left(\bar{m}_{i}\right) \geq f_{i}\left(\bar{\eta}_{i}\right)$, contradicting the last displayed inequality.

Step 3: $\bar{m}=\bar{\eta}$.

Proof: Suppose w.l.o.g. $\bar{m}>\bar{\eta}$. Then there exists $i$ such that $\bar{m}_{i}>\bar{\eta}_{i}$. Also there clearly exists $\alpha$ such that $r^{\alpha}>0$. Now, consider the first order conditions (42) and (43). Since $r^{\alpha}=s^{\alpha}$ by Step 2, RHS of (42) $\geq$ RHS of (43), which implies $f_{i}\left(\bar{\eta}_{i}\right) \geq f_{i}\left(\bar{m}_{i}\right)$. Thus $\bar{\eta}_{i} \geq \bar{m}_{i}$ since $f_{i}$ is strictly increasing by (BI). This is a contradiction.

Steps 1, 2 and 3 imply that $m=\eta$, proving Theorem 4 . 


\subsection{Proof of Theorem 9}

First we shall show

$$
m_{i}^{\alpha}>0 \Rightarrow m_{i}^{\beta}>0
$$

for all $\beta, \alpha$ such that $\xi^{\beta}(0) \leq \xi^{\alpha}(0)$. Suppose, to the contrary, that $m_{i}^{\alpha}>0$ and $m_{i}^{\beta}=0$ for some $\beta$ such that $\xi^{\beta}(0) \leq \xi^{\alpha}(0)$. The first order conditions for $\alpha$ and $\beta$ become

$$
\begin{gathered}
v_{i} \lambda_{i}\left(r_{i}^{\alpha}, \bar{m}_{i}\right)=\xi^{\alpha}\left(\bar{m}^{\alpha}\right) \\
v_{i} \lambda_{i}\left(0, \bar{m}_{i}\right) \leq \xi^{\beta}(0)
\end{gathered}
$$

where $r_{i}^{\alpha}:=m_{i}^{\alpha} / \bar{m}_{i}>0$. Since $\xi^{\alpha}\left(\bar{m}^{\alpha}\right)>\xi^{\alpha}(0) \geq \xi^{\beta}(0)$, we have $\lambda_{i}\left(r_{i}^{\alpha}, \bar{m}_{i}\right)>\lambda_{i}\left(0, \bar{m}_{i}\right)$ contradicting that $\lambda_{i}$ is increasing as in Section 2. The Theorem now easily follows.

\subsection{Proof of Theorem 11}

First we establish

Lemma 3 Assume that the utilities $U^{\alpha}$ are concave and increasing and that CVI, CVII hold. Then $U^{\alpha}\left(p\left(m^{\alpha}, m^{-\alpha}\right)\right)$ is concave in $m^{\alpha}$ for fixed $m^{-\alpha}$.

Proof of Lemma 3 Indeed, if $\lambda \in[0,1]$, if $m^{\alpha}=\lambda m^{\prime \alpha}+(1-\lambda) m^{\prime \prime \alpha}$, then CVI implies

$$
\gamma\left(m^{\alpha}, m^{-\alpha}\right) \stackrel{\alpha}{\succeq} \gamma\left(m^{\prime \alpha}, m^{-\alpha}\right)+(1-\lambda) \gamma\left(m^{\prime \prime \alpha}, m^{-\alpha}\right)
$$

Define inductively $p_{0}=p_{0}^{\prime}=p_{0}^{\prime \prime}=0 ; p_{n+1}=(1-\theta) \gamma\left(m^{\alpha}, m^{-\alpha}\right)+\theta G\left(p_{n}\right), p_{n+1}^{\prime}=(1-\theta) \gamma\left(m^{\prime \alpha}, m^{-\alpha}\right)+$ $\theta G\left(p_{n}^{\prime}\right)$ and $p_{n+1}^{\prime \prime}=(1-\theta) \gamma\left(m^{\prime \prime \alpha}, m^{-\alpha}\right)+\theta G\left(p_{n}^{\prime \prime}\right)$.

Clearly $p_{0} \stackrel{\alpha}{\succeq} \lambda p_{0}^{\prime}+(1-\lambda) p_{0}^{\prime \prime}$. Now, suppose by induction that $p_{n} \stackrel{\alpha}{\succeq} \lambda p_{n}^{\prime}+(1-\lambda) p_{n}^{\prime \prime}$. Then, since $G$ is affine and satisfies CVII , $G\left(p_{n}\right) \succeq G\left(\lambda p_{n}^{\prime}+(1-\lambda) p_{n}^{\prime \prime}\right)=\lambda G\left(p_{n}^{\prime}\right)+(1-\lambda) G\left(p_{n}^{\prime \prime}\right)$. Adding this to (44) yields $p_{n+1} \stackrel{\alpha}{\succeq} \lambda p_{n+1}^{\prime}+(1-\lambda) p_{n+1}^{\prime \prime}$.

Now observe that, as $n$ goes to $\infty, p_{n} \rightarrow p\left(m^{\alpha}, m^{-\alpha}\right), p_{n}^{\prime} \rightarrow p\left(m^{\prime \alpha}, m^{-\alpha}\right)$ and $p_{n}^{\prime \prime} \rightarrow p\left(m^{\prime \prime \alpha}, m^{-\alpha}\right)$. Therefore

$$
p\left(m^{\alpha}, m^{-\alpha}\right) \stackrel{\alpha}{\succeq} \lambda\left(m^{\prime \alpha}, m^{-\alpha}\right)+(1-\lambda) p\left(m^{\prime \prime \alpha}, m^{-\alpha}\right)
$$

In particular, $\forall i \in \mathcal{I}: p_{i}^{\alpha}\left(m^{\alpha}, m^{-\alpha}\right) \geq \lambda p_{i}^{\alpha}\left(m^{\prime \alpha}, m^{-\alpha}\right)+(1-\lambda) p_{i}^{\alpha}\left(m^{\prime \prime \alpha}, m^{-\alpha}\right)$. Since $U^{\alpha}$ just depends on $p^{\alpha}$ and is increasing and concave, we get

$$
\begin{aligned}
U^{\alpha}\left(p^{\alpha}\left(m^{\alpha}, m^{-\alpha}\right)\right) & \geq U^{\alpha}\left(\lambda p^{\alpha}\left(m^{\prime \alpha}, m^{-\alpha}\right)+(1-\lambda) p^{\alpha}\left(m^{\prime \prime \alpha}, m^{-\alpha}\right)\right) \\
& \geq \lambda U^{\alpha}\left(p^{\alpha}\left(m^{\prime \alpha}, m^{-\alpha}\right)\right)+(1-\lambda) U^{\alpha}\left(p^{\alpha}\left(m^{\prime \prime \alpha}, m^{-\alpha}\right)\right)
\end{aligned}
$$

This finishes the proof of Lemma 3 .

The existence of NE now follows exactly along the lines of Theorem 10.

\section{References}

[1] Banerji, A.; B. Dutta: "Local Network Externalities and Market Segmentation," International Journal of Industrial Organization, 27, 605-614, 2009. 
[2] Bloch, F.; N. Quérou: "Pricing in Social Network," Mimeo, 2012.

[3] Domingos, P.; M. Richardson: "Mining the network value of customers," In Proceedings of the Seventh ACM SIGKDD International Conference on Knowledge Discovery and Data Mining, pages 57-66, San Francisco, USA, August 2001.

[4] Doob, J. L.: Stochastic Processes. John Wiley \& Sons, New York, 1953.

[5] Dubey P.; R. Garg; B. De Meyer: "Competing for Customers in a Social Network: the Quasi-Linear Case," In Internet and Network Economics: Second International Workshop, WINE 2006, Patras, Greece, December 15-17, 2006, Proceedings, 2006.

[6] Hartline, J.; V. Mirrokni; M. Sundarajan: "Optimal Marketing Strategies over Social Networks," In Proceedings of $W W W$ 2008, Beijing, China, 189-198, 2008.

[7] Jackson, M.: "The economics of social networks," In Proceedings of the 9th World Congress of the Econometric Society, edited by Richard Blundell, Whitney Newey, and Torsten Persson, Cambridge University Press, July 2005.

[8] Julien, B.: "Competing in Network Industries: Divide and Conquer," Mimeo, IDEI and GREMAQ, 2001.

[9] Kempe, D.; J. Kleinberg; E. Tardos: " Maximizing the Spread of Influence through a Social Network", In Proceedings of the 9th International Conference on Knowledge Discovery and Data Mining, 137-146, 2003.

[10] Moore, C. N.: "Summability of series," The American Mathematical Monthly, 39(2):62-71, February 1932.

[11] Nash, J.: "Equilibrium points in n-person games," Proceedings of the National Academy of Science, $36: 48-49,1950$.

[12] Richardson, M.; P. Domingos: "Mining knowledge-sharing sites for viral marketing." In Proceedings of the Seventh ACM SIGKDD International Conference on Knowledge Discovery and Data Mining, pages 61-70, Edmonton, Alberta, Canada, July 2002.

[13] Saaskilahti, P.: "Monopoly Pricing of Social Goods," MPRA Paper 3526, University Library of Munich, 2007.

[14] Sahi, S.: "A note on the resolvent of a nonnegative matrix and its applications," Linear Algebra and Its Applications, 432(10):2524-2528, May 2010.

[15] Scott, J.: Social Network Analysis: A Handbook 2nd Ed. Sage Publications, London, 2000.

[16] Shapiro, C.; H. R. Varian: Information Rules: A Strategic Guide to the Network Economy. Harvard Business School Press, November 1998.

[17] Shy, O.: The Economics of Network Industries. Cambridge University Press, 2001.

[18] Tullock, Gordon. "Efficient Rent-Seeking", In Toward a Theory of the Rent-Seeking Society,Buchanan, James M., Robert D. Tollison, and Gordon Tullock, eds., College Station: Texas A \& M University Press: 97-112, 1980. 